NASA Contractor Report 178027

ICASE REPORT NO. $86-6$

NASA-CR-178027

19860014382

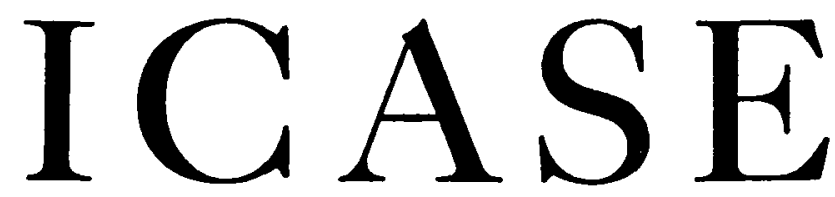

SIMULATION OF THE TURBULENT RAYLEIGH-BENARD PROBLEM

USING A SPECTRAL/FINITE DIFFERENCE TECHNIQUE

T. M. Eidson

M. Y. Hussaini

T. A. Zang

Contract Nos. NAS1-17070 and NAS1-18107

February 1986

INSTITUTE FOR COMPUTER APPLICATIONS IN SCIENCE AND ENGINEERING

NASA Langley Research Center, Hampton, Virginia 23665

Operated by the Universities Space Research Association

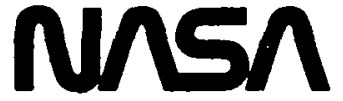

National Aeronautics and

Space Administration

Langley Research Center

Hampton, Virginia 23665

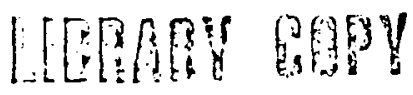

APR? 23 iY36

LANGLEY RESTAK ... VENTER

LIERRRY, NASA

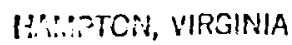





\title{
SIMULATION OF THE TURBULENT RAYLEIGH-BENARD PROBLEM \\ USING A SPECTRAL/FINITE DIFFERENCE TECHNIQUE
}

\author{
T. M. Eidson \\ Georgia Institute of Technology \\ M. Y. Hussaini \\ Institute for Computer Applications in Science and Engineering \\ T. A. Zang \\ NASA Langley Research Center
}

\begin{abstract}
The three-dimensional, incompressible Navier-Stokes and energy equations with the Boussinesq assumption have been directly simulated at a Rayleigh number of $3.8 \times 10^{5}$ and a Prandt number of 0.76 . In the vertical direction, wall boundaries were used and in the horizontal, periodic boundary conditions were applied. A spectral/finite difference numerical method was used to simulate the flow. At these conditions the flow is turbulent, and a sufficiently fine mesh was used to capture all relevant flow scales. The results of the simulation are compared to experimental data to justify the conclusion that the small scale motion was adequately resolved.
\end{abstract}

\footnotetext{
Research was supported by the National Aeronautics and Space Administration under NASA Contract Nos. NAS1-17070 and NAS1-18107 while the second author was in residence at ICASE, NASA Langley Research Center, Hampton, VA 23665-5225.
} 



\section{INTRODUCTION}

Direct simulation of turbulent fluid flows is now possible with the large vector computers that have become available $[1,2]$. Prediction of low-order flow statistics is definitely within current capabilities, and some results have already been published which show predictions of small scale turbulent features which are consistent with experimental observations [3-5]. The current study was undertaken to explore the quality of information that can be extracted from a direct flow simulation (DFS) of turbulence on a sufficiently fine mesh.

The turbulent Rayleigh-Benard problem (natural convection) was chosen for study since it is a simple turbulent flow for which a good body of experimental measurements exists. Moreover, some DFS and large-eddy simulations (LES) of this problem have been published albeit on coarser meshes. While experimental data do exist, measurements of velocity, where no mean flow exists, are difficult. Hence, there is much to be learned about turbulent natural convection from an accurate simulation.

The two requirements for conducting such a study are a high-speed computer and an efficient, accurate flow simulation code. The CYBER-205 computer with a 16 mega-word memory provides sufficient computation power. This current code has been extensively tested, and various versions of it have been used to study transition in channel flow [6]. The version used in this study includes the addition of the energy equation and a modified vertical momentum equation that includes buoyancy consistent with the Boussinesq assumption.

A simulation of a turbulent flow was then conducted, and these data as well as a discussion of the code will be presented in this paper. Though the overall goal of this work is an in-depth examination of the quality and type 
of information that can be extracted from such a simulation, the purpose of this paper is to document the basic simulation. The simulation results will be compared with experimental mean measurements as well as previous DFS results. The increased resolution of this work over previous DFS resulted in an improvement in the prediction of the Nusselt number; it was sufficiently close to experimental results to suggest that in addition to a good prediction of the large-scale flow, the small-scale features are accurately represented. Grotzbach discusses this connection extensively $[7,8]$. Comparisons with experimental data which are more dependent on the small scale components of the flow will also be presented to justify further this conclusion.

\section{RAYLEIGH-BENARD PROBLEM}

The Rayleigh-Benard problem is a simple geometry, laboratory-type problem used to study natural convection (Figure 1). Chandrasekhar [9] and Busse [10] have described the basic problem and discuss both the stability analysis and some experimental results. Krishnamurti [11,12] has summarized much experimental data and developed a map showing the qualitative flow at different values of $\mathrm{Ra}$ and $\mathrm{Pr}$, the principal independent problem parameters (defined below). For $\operatorname{Pr}=0.76$ (air) and $\mathrm{Ra}=3.8 \times 10^{5}$ the motion is turbulent, although it should possibly be qualified as low Reynolds number turbulence. Several experimental studies [13-18] and numerical simulations $[7,19,20]$ (both LES and DFS) have been completed in the qualitatively similar Pr-Ra region. The flow at these values of $\operatorname{Pr}$ and $\mathrm{Ra}$ consists of a core flow (a horizontal layer in the middle $80 \%$ of the fluid layer) and a boundary region near each plate. The turbulence is statistically homogeneous in the 
horizontal directions for both layers. In the core the vertical variation of most statistical quantities is small. In the boundary layer there is a transition from molecular dominated physical processes near the wall to the fully turbulent core flow.

This flow is described by the incompressible Navier-Stokes equations modified to include the effect of temperature-induced density variations on the buoyancy force (Boussinesq assumption) plus the temperature equation. These equations, when non-dimensionalized by $\alpha, h$, and $\Delta T$, are

$$
\begin{aligned}
& \frac{\partial u_{i}}{\partial t}+\frac{\partial\left(u_{i} u_{j}\right)}{\partial x_{j}}=-\frac{\partial P}{\partial x_{i}}+\operatorname{Pr} \frac{\partial^{2} u_{i}}{\partial x_{j}^{2}}+\operatorname{Pr} \operatorname{Ra} T \delta_{i 3}, \\
& \frac{\partial T}{\partial t}+\frac{\partial\left(T u_{j}\right)}{\partial x_{j}}=\frac{\partial^{2} T}{\partial x_{j}^{2}}+u_{3},
\end{aligned}
$$

and

$$
\frac{\partial u_{j}}{\partial x_{j}}=0
$$

The temperature and pressure in Equations $(1 \mathrm{a}, \mathrm{b}, \mathrm{c})$ are the difference between the actual temperature, $T_{a}$, and pressure, $P_{a}$, and the values due to the static temperature gradient only. These are defined as follows:

$$
\begin{aligned}
T_{a}(\underline{x}, t) & =T_{0}-x_{3}+T(\underline{x}, t) \\
& =T_{0}+T_{r}(\underline{x}, t)
\end{aligned}
$$

and 


$$
\frac{\partial P(x, t)}{\partial x_{3}}=-\frac{g h^{3}}{\alpha^{2}}-\operatorname{Pr} \operatorname{Ra} x_{3}+\frac{\partial P(x, t)}{\partial x_{3}} .
$$

The dependent variables in the problem are the velocity components, $u_{i}$ or $(u, v, w)$, the temperature, $T$, and the fluid pressure, $P$. The independent variables are the spacial coordinates, $x_{i}$ or $\underline{x}=(x, y, z)$, and time, $t$. The indices $i=1$ and $i=2$ signify the horizontal directions, and $i=3$ denotes the vertical direction. The problem parameters are the thermal diffusivity, $\alpha$; the kinematic viscosity, $\nu$; the acceleration of gravity, $g$; the reference fluid density, $\rho_{0}$; the coefficient of thermal expansion, $\beta$; a reference temperature (the temperature of the lower plate), $\mathrm{T}_{0}$; and the temperature difference between the two plates, $\Delta T$. The dependent and independent variables have been non-dimensionalized by $\alpha / \mathrm{h}$ (velocity), $\Delta \mathrm{T}$ (temperature), $\rho_{0} \alpha^{2} / h^{2}$ (pressure), $h$ (coordinates), and $h^{2} / \alpha$ (time). The Rayleigh number, $\mathrm{Ra}=\frac{\mathrm{g} \beta \Delta \mathrm{Th}^{3}}{\nu \alpha}$, and Prandlt number, $\operatorname{Pr}=\nu / \alpha$, are the principal non-dimensional problem parameters.

\section{NUMERICAL METHOD}

The Fourier finite difference algorithm developed by Moin and Kim [21] for their large-eddy simulations of turbulent channel flow has been applied, with modifications, to the present direct simulation of turbulent RayleighBenard flow. This Rayleigh-Benard Fourier-finite difference Method (RBFFDM) is an unsplit method on a grid staggered (for the pressure variable and the continuity equation) in the vertical ( $z$ ) direction. Fourier collocation is used for the spacial discretization in the $x$ and $y$ directions whereas in the vertical direction second-order finite-differences are employed on the 
non-uniform grid,

$$
z_{k}=\left(1-\cos \left(k \pi / N_{z}\right)\right) / 2, \quad k=0,1, \cdots, N_{z}
$$

The time discretization is Crank-Nicolson for the viscous and conductive terms and backward Euler for the pressure gradient term. The advection and buoyancy terms are handled by a third-order Adams-Bashforth method.

The implicit part of the algorithm requires, for each pair of horizontal Fourier wavenumbers, the solution of 2 real, block tridiagonal systems (involving the velocities and pressure) and, Independently, 2 real, scalar tridiagonal systems (for the temperature). The block-tridiagonal equations were scaled as described by Zang \& Hussaini [6] for their Fourier-Chebyshev version of the corresponding channel flow algorithm. Pivoting has proven to be unnecessary for this system. The block-tridiagonal solution algorithm takes advantage of the many zero elements which occur. Vectorization of this phase of the algorithm is achieved by solving for many pairs of Fourier wavenumbers at the same time.

Equations $(1 \mathrm{a}, \mathrm{b}, \mathrm{c})$ were solved on the region, $0 \leqslant x<A_{x}, 0<y<A_{y}$, $0<z<1$. Under the present scaling of the vertical direction, the lengths $A_{x}$ and $A_{y}$ correspond to the aspect ratios of the two horizontal directions to the vertical one. The boundary conditions at the lower and upper walls, $z=0$ and $z=1$, are the conventional no-slip and no temperature jump conditions. In the horizontal directions, periodic boundary conditions are assumed. These aspect ratios must be large for reasonable correspondence with experiments. The aspect ratios are related to the resolution by $A_{x}=N_{x} \Delta x$ and $A_{y}=N_{y} \Delta y$. The computer memory limitation places an upper bound on 
$N=N_{x} N_{y} N_{z}$; therefore, to have a large $A_{x_{1}}$ along with sufficiently small $\Delta x_{i}$, some compromise is necessary. The values chosen, $A_{x}=4$ and $A_{y}=2$, allow the available computer memory to be used for better small scale resolution. This will be further discussed in Section IV.

The RBFFDM code has been implemented on a CDC Cyber 205 with 2 pipes and 16 million 64 -bit words of main memory. For each grid point 13 variables were stored. Additional storage equivalent to 7 variables per grid point was used to facilitate vectorization. A total of 11 million words was used for the simulation on the $128 \times 64 \times 64$ grid. Vector lengths for the explicit portion of the algorithm were between 4 and 40 thousand. Typically, one-fourth of the implicit equations were solved together. The vector lengths here were roughly 1,000. The linked triad feature was heavily exploited. A single time-step required 6.8 seconds of $\mathrm{CPU}$ time and no $I / 0$ time since the job was run entirely within the central memory. The sustained speed of the calculation was 100 MFLOPS.

The start-up phase of the calculation took 2100 time-steps and the data collection an additional 5600 steps. A total of 12 hours of CPU time was required for the data collection. This includes the time for some preliminary diagnostics. The Courant number, defined as the maximum over the grid of the quantity

$$
\left[\frac{|u|}{\Delta x}+\frac{|v|}{\Delta y}+\frac{|w|}{\Delta z}\right] \Delta t,
$$

ranged between 0.19 and 0.26 and averaged 0.23 for the data collection phase. 


\section{COMPARISON WITH PREVIOUS WORK}

The turbulent flow of the Rayleigh-Benard problem is assumed to be homogeneous in both horizontal directions as well as statistically steady in time (after a start-up period). Experimental data are usually presented using some combination of a long-time average as well as a spacial average in one or both horizontal directions. The simulation results presented as horizontal averages, $\langle>$, have been averaged over both the $x$ and $y$ directions (except for the 1-D $x$-spectra which were averaged in $y$ only). In addition, they have been time averaged over a time period equal to $10 / \mathrm{W}_{c}$, where $W_{c}=(N u \operatorname{Pr} R a)^{1 / 3}$. (See Deardorff [22] for a discussion of $W_{c}$, the scaling velocity for the large eddies.) This perlod, which should consist of several large eddy turn-over times, was found adequate by Eidson [19]. Volume averages were time averaged as well. All the simulation results presented below are both horizontally and temporally averaged unless otherwise specified.

Prediction of the average vertical heat flux, the Nusselt number in nondimensional form, is an important result of any natural convection study. Previous simulations have predicted values of the Nusselt number, $\mathrm{Nu}$, which are slightly higher than those measured experimentally. Grotzbach [8] has discussed extensively this discrepancy and has shown that inadequate resolution is partially responsible. In Table 1 , the results from both simulations and experiments are shown. The prediction of the present study lies at the the upper range of experimental measurements and below the Nusselt numbers predicted by previous, coarser-grid simulations. In the present simulation, $\mathrm{Nu}$ was calculated at each $\mathrm{z}$ level. The average value is reported in Table $I$. The variation with $z$ was small (approximately \pm 0.1 ) except very near the lower wall where $\mathrm{Nu}$ increased to 7.0 . 
The aspect ratio of the horizontal to vertical boundary lengths also is known to affect $\mathrm{Nu}[8,15]$. The values of $A_{x}=4$ and $A_{y}=2$ are smaller than the values of 4 to 7 suggested by experimentalists as the minimum for removing significant side boundary effects. Although the aspect ratlo effect is not negligible, especially on Nusselt number [8], a large aspect ratio was foregone in favor of better small scale resolution in view of the goal of this study: to resolve eddies down to nearly the dissipation scales.

The relative temperature, $\mathrm{T}_{\mathrm{r}}$, vertical profile is compared to data of studies by Chu and Goldstein [16] in Figure 2. A line with slope, $\frac{\partial T_{r}}{\partial z}=N u$, is drawn in this figure. From this one can estimate the conductive layer thickness, $\delta_{c}$. This will be used later in a more extensive examination of the temperature data near the wal1.

In Figures 3 and 4 , the vertical dependence of the velocity and temperature RMS values are compared to the simulation of Grotzbach [7] and the measurements of Deardorff and Willis [13] (slightly larger Ra). In Table 1 a comparison for the centerline values is presented for a wide range of studies. The present improved resolution reduces slightly the predicted levels of the RMS temperature over previous simulations. However, no systematic change in the RMS velocity levels with the improved spacial resolution was observed. Both trends are consistent with Grotzbach's results. Considering the variation in experimental values and the uncertainty in aspect ratio effects, the results appear quite satisfactory.

The wT correlation coefficient, $\mathrm{C}(\mathrm{wT})$, is constant for $0.2<\mathrm{z}<0.8$, giving a value of 0.71 (Figure 5). Both Grotzbach [7] and Eidson [20] previously obtained a value of approximately 0.67 . Deardorff and Willis measured approximately 0.60 for a slightly higher Ra, but C(wT) should 
decrease with increasing Ra. Near the wall all four studies differ. Deardorff and Willis measured a significant drop as the wall was approached, but warned that since the numerator and denominator of $C(w T)$ become small, their results are uncertain. The increase in $C(w T)$ near the wall found in the current study was not observed in either previous simulation, but neither of these had sufficient resolution in this region. Note that only the data of Grotzbach's case 9 with 16 vertical grid points was available for comparison. Also, horizontal averages of the uv, uw and vw correlations were calculated. They were all approximately zero as would be expected for turbulence homogeneous in the horizontal directions.

In Figure 6, the several terms in the horizontally averaged kinetic energy equation are plotted versus $z$ and compared with experimental data at a slightly larger value of $\mathrm{Ra}$. These terms are

$$
\begin{aligned}
& \frac{\partial\langle E\rangle}{\partial t}= \operatorname{Pr} \operatorname{Ra}\left\langle w T_{a}\right\rangle-\frac{\partial}{\partial z}\left\langle w\left(E+P_{a}\right)\right\rangle \\
& \text { Production } \quad \text { Diffusion }
\end{aligned}
$$

where

$$
E=\frac{1}{2} u_{i} u_{i}
$$

Since the production term in the core is equal to ( $\mathrm{Pr} \mathrm{Ra} \mathrm{Nu}$ ), the experimental data of Deardorff \& Willis [13] at $\mathrm{Ra}=6.3 \times 10^{5}$ and $\mathrm{Nu}=5.7$ would be expected to be about $40 \%$ higher than the current simulation. For 
clarity, only a few key values from Deardorff and Willis are shown to demonstrate the general agreement between experiment and simulation. The four terms are all essentially constant and approximately $40 \%$ below the experimental data for the core region. In the boundary region the variation with $z$ is similar for both experiment and simulation. The diffusion term (for the experiment and simulation) peaks at $a \quad z$ value of 0.035 , and the molecular diffusion term of the simulation peaks at a slightly higher $z$. The experimental point plotted at a low $z$ value is at these peaks for these two terms. Actually, one would expect the peaks for the lower $\mathrm{Ra}$ to be at a slightly larger $z$ since the boundary region thins with increasing Ra. From a closer inspection of the data, the molecular dissipation also can be seen to have a small positive value between $z=0.1$ and $z=0.2$ as was found by Deardorff and Willis. The production and dissipation terms have the same general shape in both studies. The volume averages of the production and dissipation terms were $1.641 \times 10^{6}$ and $1.637 \times 10^{6}$, respectively. Also, the volume average of the molecular transfer term was $9.0 \times 10^{3}$.

One advantage of the simulation technique is that quantities which are experimentally difficult to measure can be easily calculated. Figures 7 to 9 give three examples. In Figure 7 the absolute value of each of the three components of vorticity is shown. As expected, the $x$ and $y$ components are large near the wall due to the creation there of a boundary layer by the large eddies. Near the center the flow is more isotropic. The ratio of the volume average of the horizontal to vertical vorticity was 5.0 and 4.3 for the $x$ and $y$. directions, respectively. This is higher by a factor of 2 than in the previous DFS by Eidson [19]. Figure 8 shows that the only significant velocity skewness is for the $w$ component near the wall. A negative value 
for the $w$ component is reasonable since the fluid particles with negative velocity (near the lower wall) come from the core where there is more velocity variation due to the turbulent cascading process. The particles with a positive velocity originate near the wall where the motion is damped and they have a more uniform value. Recall that the horizontal average of the w velocity equals zero. The flatness profile of the velocities is shown in Figure 9. For the horizontal components, these approach 3 in the core, a value which is similar to that in channel flow turbulence away from the wal1 [21]. The larger flatness factor near the wall suggests that the flow is more intermittent in this region.

The wT cross-spectra for several $z$ values are plotted in Figures 10 to 13. These are 1-D spectra in the $x$-direction. They have been normalized so that they integrate to $\langle w T\rangle$. The wavenumber, $k$, is defined such that $2 \pi k x$ is the argument of the periodic expansion functions. Notice that the spectra decrease with $\kappa$ except near the wall where they are roughly constant with k. This trend was found also in the $u, w$ and $T$ spectra but not in $v$. Near the center the most energy would be expected in the largest available scales for the small values of A in this study. Near the wall, smaller horizontal structures would be expected, and thus significant energy at a wavenumber greater than the minimum is a reasonable result. Since this is low Ra turbulence, an equilibrium region would not be expected, and indeed none was found here. The drop-off with wavenumber is rapid due to the importance of viscous effects at all the scales.

To evaluate the simulation further, a more sensitive measure of the small scale features of the flow field, especially in the wall region, was sought. Carroll [14] has made direct instantaneous measurements of the 
temperature and the vertical temperature gradient and calculated the mean, standard deviation, and skewness. Carrol1's data are compared with the results of this simulation in Figures 14 to 20 . The data of Carroll in these figures are a mean curve (drawn by Carroll) through the data points. The bars show the range of data scatter. The mean of the relative temperature (simulation data shown in Figure 2) and its vertical derivative (Figure 18) show good agreement with Carroll's data. The higher order statistics give a variation with $z$ which is similar to the experimental results although the magnitudes differ in some cases. In Table 1 , a significant variation in experimental measurements of the RMS of $T_{r}, R\left(T_{r}\right)$, between different experimental studies is apparent. Carroll used a stationary probe, and this may have reduced the fluctuations coming from the direction of the probe and lowered the statistical levels. Also Carroll measured the same RMS temperature level for $\mathrm{Nu}$ ranging from 5.5 to 14.0 . Both experiments $[13,15]$ and simulations [20] have shown that $R\left(T_{r}\right)$ increases with decreasing Nu (or $\mathrm{Ra}$ ). Since the magnitude of the data is in question in some cases, the conclusions drawn from the simulation/experiment comparison are based mainly on changes of slope with vertical distance from the wall. These variations correspond to differences in the dominant physics in the different layers, and the simulation was able to predict these layers in agreement with the experimental results.

A four-layer (or three-layer with the middle divided into 2 sub-1ayers) model is consistent with both the experimental and simulation results. These are: 
$\begin{array}{lc}\text { (i) a conduction dominated layer } & 0<\mathrm{Nu} *_{2}<\delta_{1}{ }^{*} \\ & 0.1<\delta_{1}^{*}<0.3 \\ & \\ \text { (ii) an inner boundary layer } & \delta_{1}{ }^{*}<\mathrm{Nu}_{z}^{*}<\delta_{2}{ }^{*} \\ & \delta_{2}{ }^{*} \approx 0.5 \\ \text { (iii) an outer boundary layer } & \delta_{2}{ }^{*}<\mathrm{Nu}{ }^{*}<\delta_{3}{ }^{*} \\ & \delta_{3}{ }^{*} \approx 1.5 \\ \text { (iv) the core } & \delta_{3}{ }^{*}<\mathrm{Nu}{ }^{*}<\mathrm{Nu} / 2\end{array}$

These layers are all well resolved by the simulation as they contain $8,4,9$, and 11 grid points respectively (assuming $\delta_{1}^{*}=0.2$ ).

Determination of $\delta_{1}{ }^{*}$ is uncertain. As was mentioned previously (Figure 2), divergence of the temperature profile from a straight line with a normalized slope equal to $\mathrm{Nu}$ (the temperature gradient at the wall) gives $\delta_{1}{ }^{*}=\delta_{c}^{*}=0.26$. Another measure is that the wT correlation rises from $1 \%$ to $10 \%$ of 1 ts core value near $\mathrm{Nu}^{*} \mathrm{z} \approx 0.1$.

The inner boundary layer is characterized by the transition from molecular to turbulent dominated processes. Both experiment and simulation show that $\frac{\partial T_{r}}{\partial z}$ has been reduced by $50 \%$ between $\mathrm{Nu}_{z}$ of 0.4 and 0.5 (Figure 18). In the inner boundary layer, $\mathrm{R}\left(\mathrm{T}_{\mathrm{r}}\right)$ reaches a maximum (Figure 14), the skewness factor for $T_{r}, S\left(T_{r}\right)$, changes sign (Figure 16), and the flatness factor for $T_{r}, F\left(T_{r}\right)$, reaches a minimum (Figure 17). The skewness, $S\left(T_{r}\right)$, continues to increase reaching a positive maximum in the outer boundary layer. The above trends, shown in both studies, suggest significant changes in the flow character in these layers. 
In the outer layer, the flow transitions to a core region where the large eddies (the most efficient heat transfer agents) carry the heated fluid from the bottom to the top. At $\mathrm{Nu}_{\mathrm{z}} \approx 1.5$, the temperature gradient, $\frac{\partial \mathrm{T}_{r}}{\partial z}$, has been reduced to $1 \%$ of its maximum value (Figure 18). The skewness, $S\left(\mathrm{~T}_{\mathrm{r}}\right)$, decreases in the outer layer and approaches zero near the center of the core (Figure 16). Also note, in the outer layer $R\left(T_{r}\right)$ vartes as $z^{-1 / 3}$ compared with $z^{+1}$ in the inner layer, a trend which is found in both studies (Figure 15). The simulation predicts a transition to a constant level for $R\left(T_{r}\right)$ in the center in agreement with Deardorff and Willis [13] (Figure 4). However, Carrol1's data suggest that $R\left(T_{r}\right)$ varies as $z^{-1 / 3}$ in the core as well as the outer layer.

A possible discrepancy between the simulation results and Carrol1's data is seen in the $R\left(\frac{\partial T_{r}}{\partial z}\right)$ plot (Figure 19). At values of $\mathrm{Nu}^{*} z$ between 0.4 and 1.0 the simulation shows a hump. However, Carrol1's choice of an "average curve" has significant scatter only on top of the curve. Re-drawing the data fit through this scatter would result in a similar hump in the outer boundary layer region. Another example, where the simulation results suggest that the "averaged curve" through sparse, scattered data is not correct, is Carrol1's choice for the temperature skewness profile for $N u *_{z}<0.1$ (Figure 16). He suggests that the curve should continue decreasing to $S\left(T_{r}\right)=-1.5$ and then rise to zero at $\mathrm{Nu}^{*} \mathrm{z} \approx 0.01$. However, only a few data points which gave significant scatter were measured in this region. From an examination of the data in Carro11's paper, it is not inconsistent to draw the experimental data fit to turn between $\mathrm{Nu}^{*} \mathrm{z}=0.1$ and 0.2 and be parallel to the simulation results somewhere between the dashed lines shown in Figure 16 . One discrepancy that cannot be explained by data scatter is the comparison between 
studies of $\mathrm{S}\left(\frac{\partial \mathrm{T} r}{\partial z}\right)$ away from the wall. The simulation predicts that $\mathrm{S}\left(\frac{\partial \mathrm{T} r}{\partial z}\right)$ returns to zero in the outer layer and remains zero in the core (Figure 20). However, the experiments show a return to negative skewness in the core.

The final comparison is for the temperature gradient versus $\mathrm{Nu}_{\mathrm{z}}$ near the wall (Figure 18). Various power laws have been hypothesized which predict a linear slope for the data plotted in log-log form. Carroll [14], Businger [23], and Monin and Yaglom [24] discuss the various theories. Carrol1's data show that only for the range $0.4<\mathrm{Nu}_{\mathrm{z}}<1.0$ does a simple power law seem reasonable and even then the data have a slow change in slope in this region. The current simulation. fits Carroll's data nicely and thus also predicts a slope of -2 in this range of $z$. The value of -2 is predicted by the theory of Malkus [25].

From these comparisons, it can be seen that the simulation data represent a turbulent realization which has a good agreement with experimental, averaged measurements. The agreement, at least in prediction of sub-layers using the variance and skewness data, shows that the small scale turbulent features are accurately represented near the wall. This is important because a complete understanding of the Rayleigh-Benard convection requires studying the thin boundary layer which exists as a result of the large scale motion in the core. From isothermal, flat plate boundary layer studies, it is known that significant small scale events occur in this type of region which are important to the global flow.

Some of the features that we have observed in this calculation were also found in a reference pointed out to us by a referee [26]. However, we have not yet performed a detailed comparison with this data. 


\section{CONCLUDING REMARKS}

From the comparison with the gross properties of the experimental data, it can be concluded that the completed computer simulation is a reasonably accurate realization of a turbulent flow even down to the dissipation scales. Moreover, the detailed database has enabled us to identify a feature in the skewness of the vertical temperature derivative which has been overlooked in the experiment due to data scatter.

Obviously, a far more detailed analysis of the simulation results is in order. This simulation is of sufficient accuracy to study the small scale structure of a natural convection flow in a similar manner to the work previously done for channel flows [4,21]. Moreover, it can be used to help interpret experimental measurements which are typically limited to a few spacial points and fewer than all three velocity components. Finally, we feel this database can be used to evaluate turbulent models and other theoretical ideas.

\section{Acknowledgment}

The authors would like to express their appreciation to Control Data Corporation for the use of a CYBER-205 at the Arden Hills Plant in Minnesota during the initial phase of this study, and to J. Lambiotte and G. Tennille for their assistance in running the code. 


\section{References}

1. Rogallo, R. S. and Moin, P. [1984] Numerical simulation of turbulent flows. Ann. Rev. F1. Mech., 16, 99-137.

2. Schumann, U., Grotzbach, G. and Kleiser, L. [1980] Direct numerical simulation of turbulence. Prediction Methods for Turbulent Flows, Hemisphere Publ. Corp., (W. Kollman, ed.), 124.

3. Brachet, M. E., et al., [1983] Smal1-scale structure of the TaylorGreen vortex. J. Fluid Mech., 130, 411-453.

4. Moin, P. and Kim, J. [1985] The structure of vorticity field in turbulent channel flow. Part 2. Study of ensemble-averaged fields. J. Fluid Mech. To appear.

5. Moser, R. D. and Moin, P. [1984] Direct numerical simulation of curved turbulent channel flow. NASA TM 85974. Also Report TF-20, Department of Mechanica1 Engineering, Stanford University.

6. Zang, T. A., and Hussaini, M. Y. [1985] Numerical experiments on . subcritical transition mechanisms. AIAA Paper 85-0296.

7. Grotzbach, G. [1982] Direct numerical simulation of laminar and turbulent Benard convection. J. Fluid Mech. 119, 27. 
8. Grotzbach, G. [1983] Spacial resolution for direct numerical simulation of the Rayleigh-Benard convection. J. Comp. Phys. 49, 241.

9. Chandrasekhar, S. [1961] Hydrodynamic and Hydromagnetic Stability. Clarendon Press, Oxford.

10. Busse, F. H. 1981 Transition to turbulence in Rayleigh-Benard convection. Topics in Applied Physics. Vol. 45: Hydrodynamic Instabilities and the Transition to Turbulence. (H. L. Swinney \& J. P. Gollub, eds.), Springer-Verlag.

11. Krishnamurti, R. [1970a] On the transition to turbulent convection. Part 1. The transition from two- to three-dimensional flow. J. Fluid Mech. 42, 295.

12. Krishnamurti, R. [1970b] On the transition to turbulent convection. Part 2. The transition to time-dependent flow. J. Fluid Mech. 42, 309.

13. Deardorff, J. W., and G. E. Willis [1967] Investigation of turbulent thermal convection between horizontal plates. J. Fluid Mech. 28, 675 .

14. Carro11, J. J. [1976] The thermal structure of turbulent convection. J. Atmos. Sc. 33, 642 .

15. Fitzjarrald, D. E. [1976] An experimental study of turbulent convection in air. J. Fluid Mech. 73, 693. 
16. Chu, T. Y. and R. J. Goldstein [1973] Turbulent convection in a horizontal layer of water. J. Fluid Mech. 60, 1, 141.

17. Goldstein, R. J. and T. Y. Chu [1969] Thermal convection in a horlzontal layer of air. Prog. Heat and Mass Transfer. 2, 55.

18. Denton, R. A. and I. R. Wood [1979] Turbulent convection between two horizontal plates. Int. J. Heat Mass Transfer. 22, 1339.

19. Eidson, T. M. [1982] Numerical simulation of the turbulent RayleighBenard problem using subgrid modeling. Ph.D. Thesis, University of Michigan.

20. Eidson, T. M. [1985] Numerical simulation of the turbulent RayleighBenard problem using subgrid modeling. J. Fluid Mech. 158, 245.

21. Moin, P. and Kim, J. [1982] Numerical investigation of turbulent channel flow. J. Fluid Mech. 118, 341 .

22. Deardorff, J.W. [1970] Convection velocity and temperature scales for the unstable planetary boundary layer and for Rayleigh convection. J. Atmos. Sci. 27, 1211 .

23. Businger, J. A. [1973] Turbulent transfer in the atmospheric surface layer. Workshop in Micrometeorology, American Meteorology Society, 67. 
[24] Monin, A. S. and Yaglom, A. M. [1971] Statistical Fluid Mechanics, 1 MIT Press.

[25] Malkus, W. V. R. [1954] The heat transport of thermal turbulence. Proc. Roy. Soc. (London), A. 225196.

[26] Grotzbach, G. [1980] Über das räumliche Auflösungsvermogen numerischer Simulationen von turbulenter Benard-Konvektion. Kernforschungszentrum Karlsruhe. 2981B 
Table 1. Horizontal Averages at the Vertical Center

\begin{tabular}{|c|c|c|c|c|c|c|}
\hline Direct Simulation & Grid & $\mathrm{Ra}$ & $\mathrm{Nu}$ & $\mathbf{u}_{\mathrm{RMS}}$ & $\mathrm{w}_{\text {RMS }}$ & $\mathrm{T}_{\mathrm{r}_{\mathrm{RMS}}}$ \\
\hline Current Study & $128 \times 64 \times 64$ & $3.8 \times 10^{5}$ & 6.6 & 52 & 116 & 0.08 \\
\hline Grotzbach* & & $3.8 \times 10^{5}$ & & & & \\
\hline$(\operatorname{case} 7)$ & $16 \times 16 \times 16$ & & 7.8 & -- & 109 & - \\
\hline (case 9) & $32 \times 16 \times 16$ & & 7.4 & 50 & 107 & 0.08 \\
\hline (case 14) & $64 \times 32 \times 32$ & & 6.9 & - & 109 & 0.08 \\
\hline Eidson & $64 \times 64 \times 16$ & $3.8 \times 10^{5}$ & 8.1 & 70 & 112 & 0.11 \\
\hline \multicolumn{7}{|l|}{ Experiment } \\
\hline Deardorff \& Willis & & $6.8 \times 10^{5}$ & 5.8 & 65 & 120 & 0.08 \\
\hline Carroll & & $3.8 \times 10^{5}$ & $5.9 *$ & - & -- & $0.05 *$ \\
\hline Fitzjarrold & & $3.8 \times 10^{5}$ & $6.2 *$ & $80 *$ & $120 *$ & $0.05 *$ \\
\hline Chu \& Goldstein & & $3.8 \times 10^{5}$ & $6.5 *$ & - & -- & $\cdots$ \\
\hline Goldstein \& Chu & & $3.8 \times 10^{5}$ & $5.4 *$ & - & - & -- \\
\hline
\end{tabular}

*Calculated from curve fit of data over a range of Ra. 

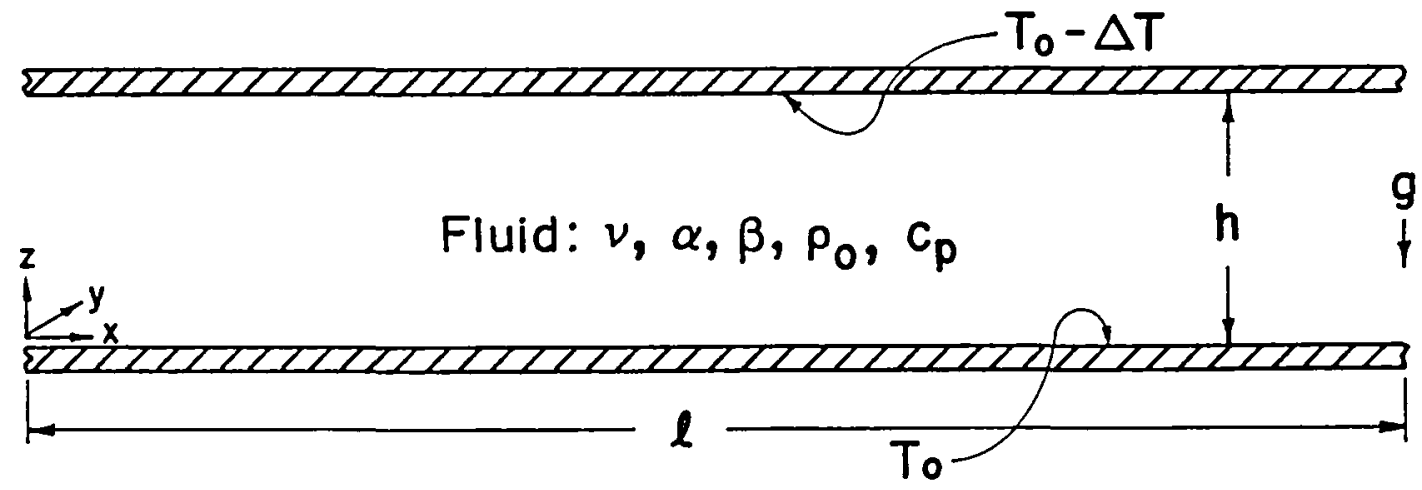

Figure 1. Geometry and Parameters of the Rayleigh-Benard Problem 


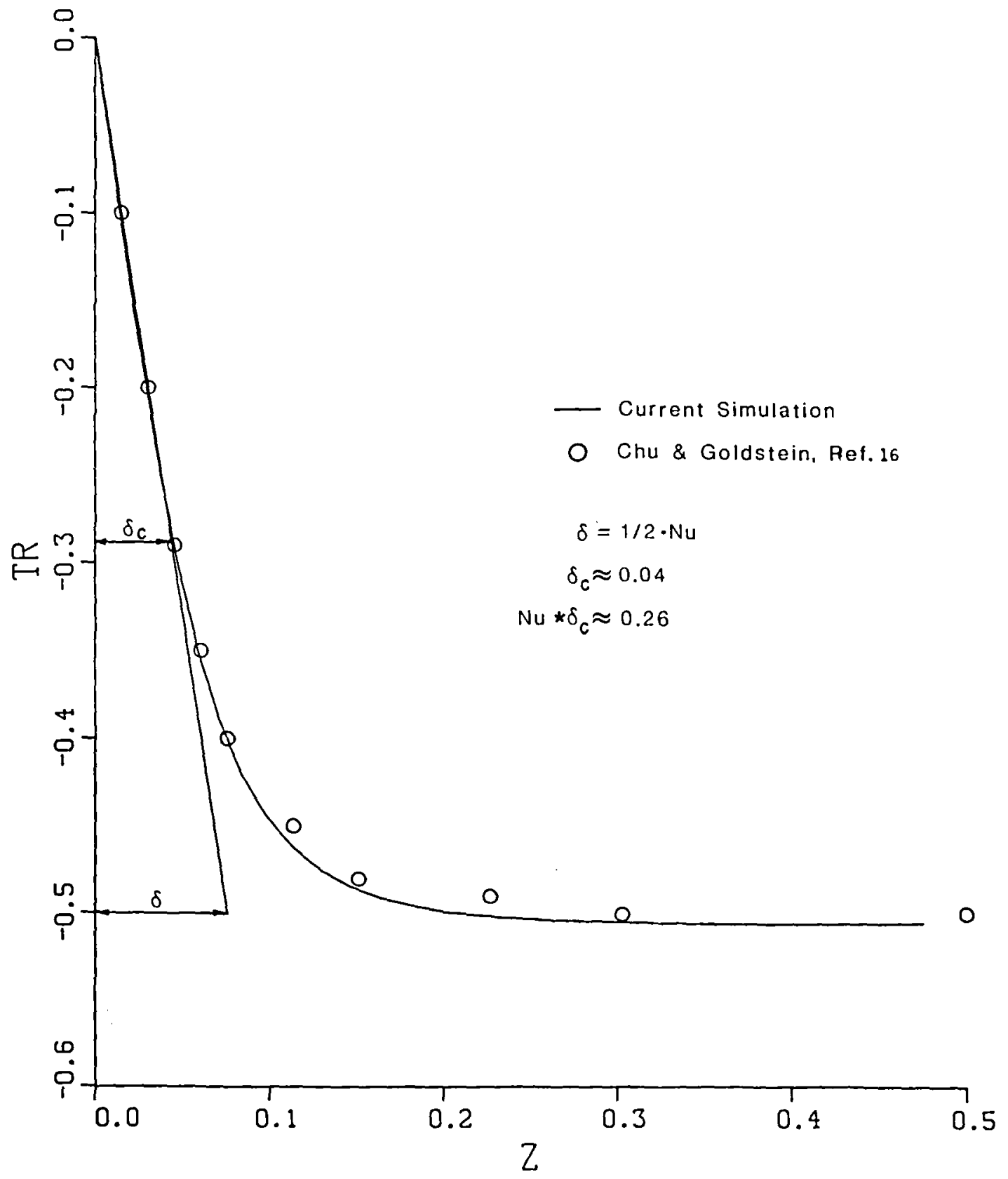

Figure 2. Estimation of the region for which the relative temperature varies linearly with $z$. 


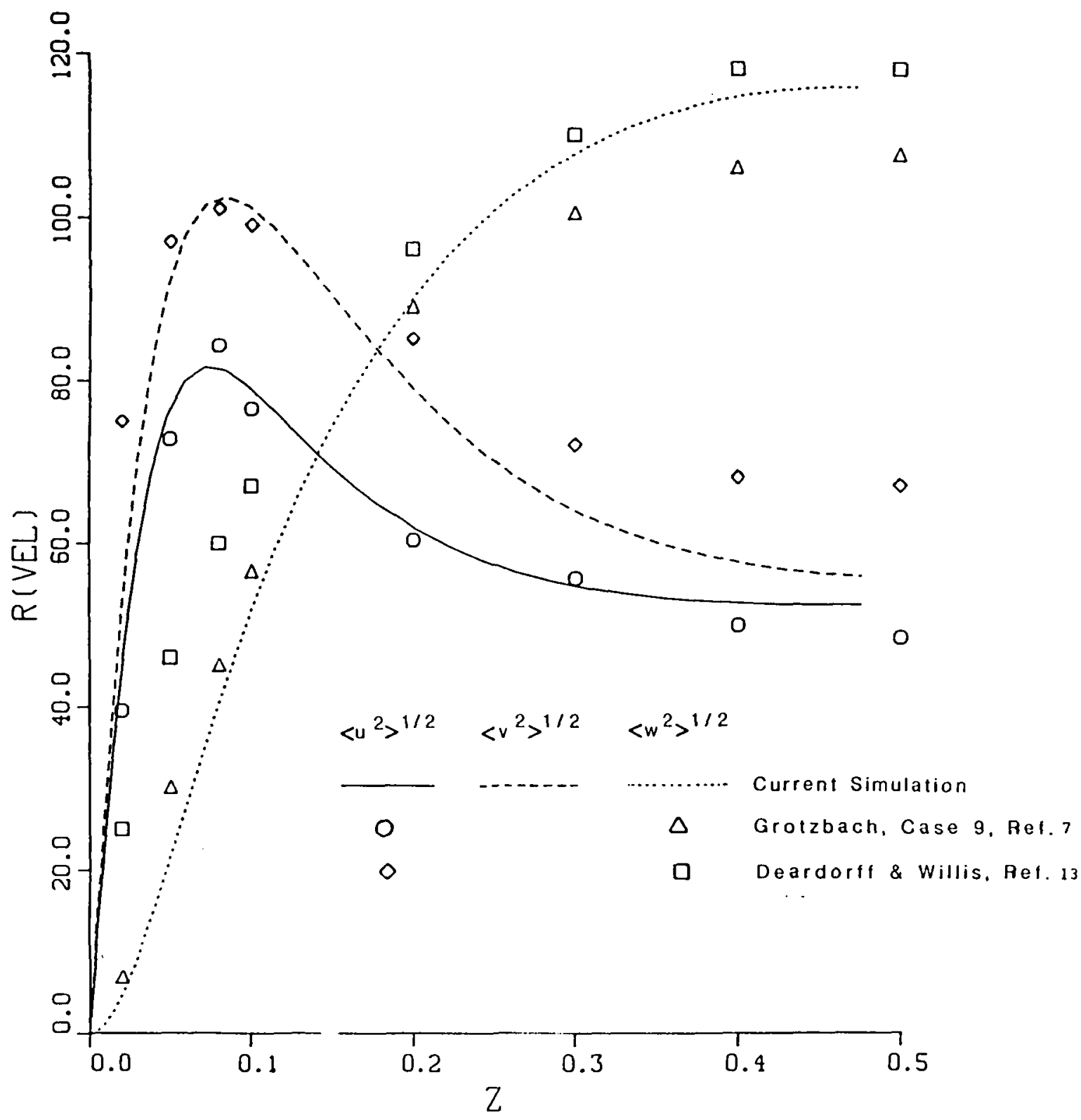

Figure 3. Comparison with experiments and previous

simulations of the RMS of the velocity components 


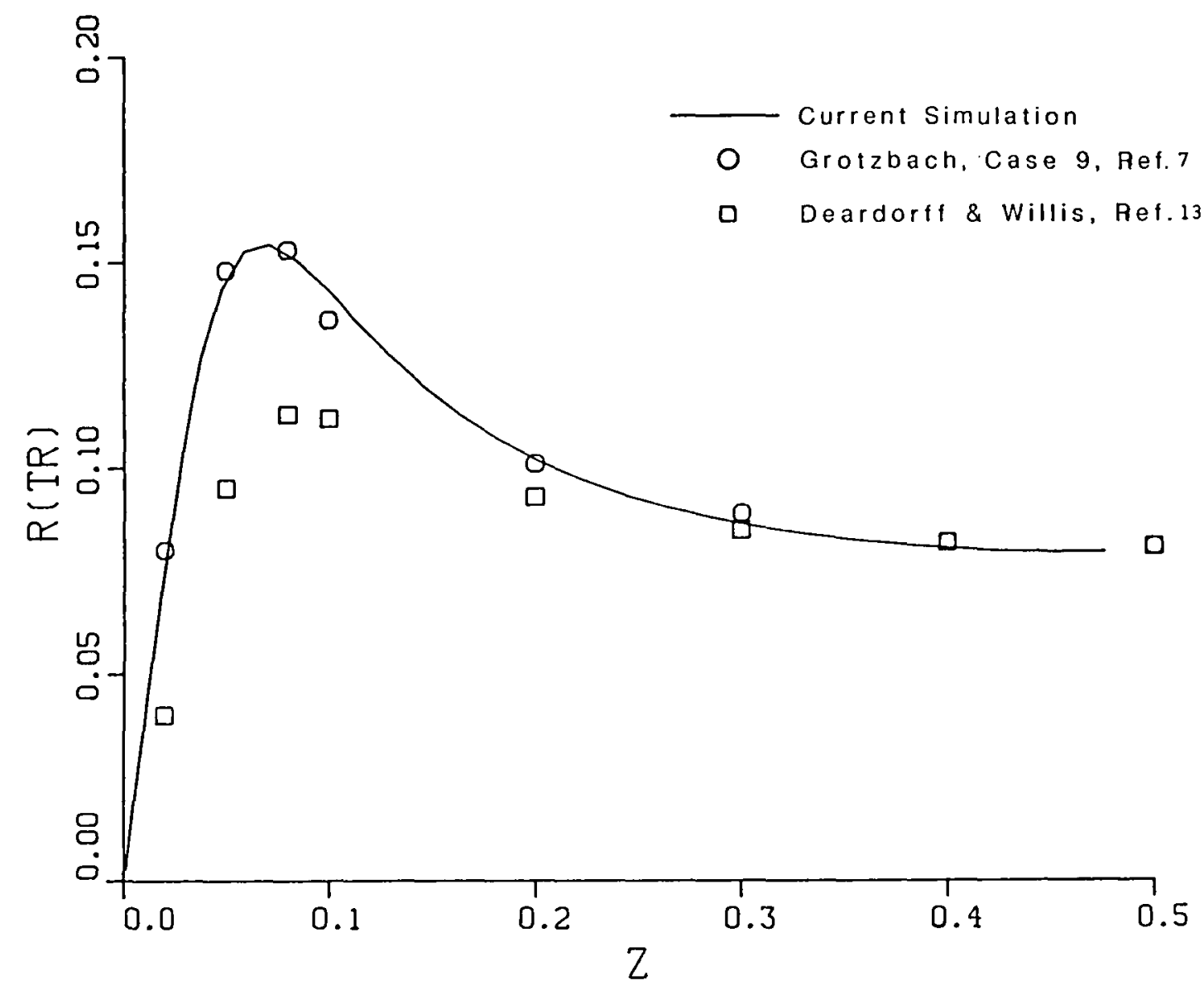

u

Figure 4. Comparison with experiments and previous simulations of the RMS of relative temperature. 


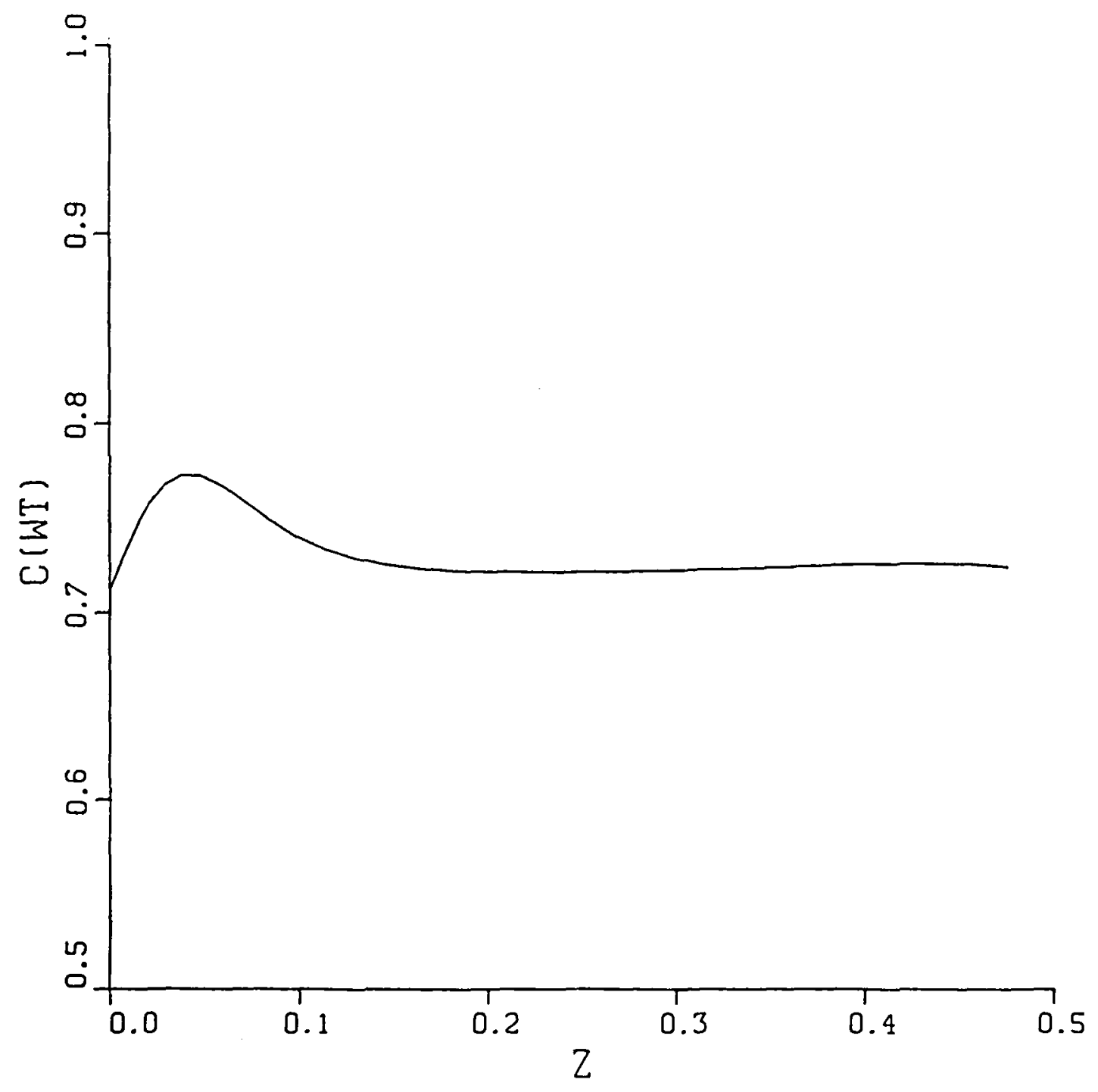

Figure 5. wT correlation versus height. 


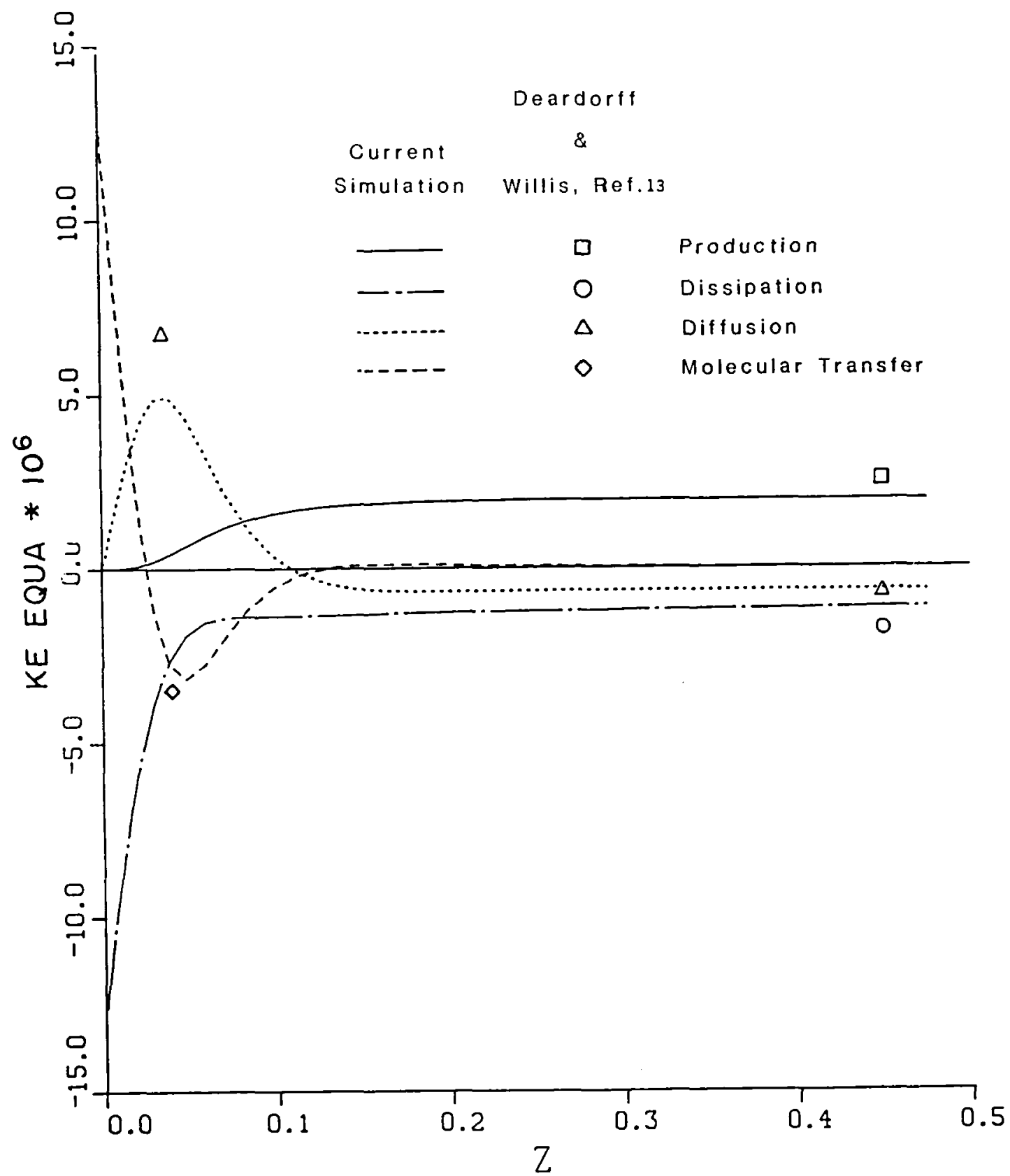

Figure 6. Terms in the kinetic energy equation versus height. 


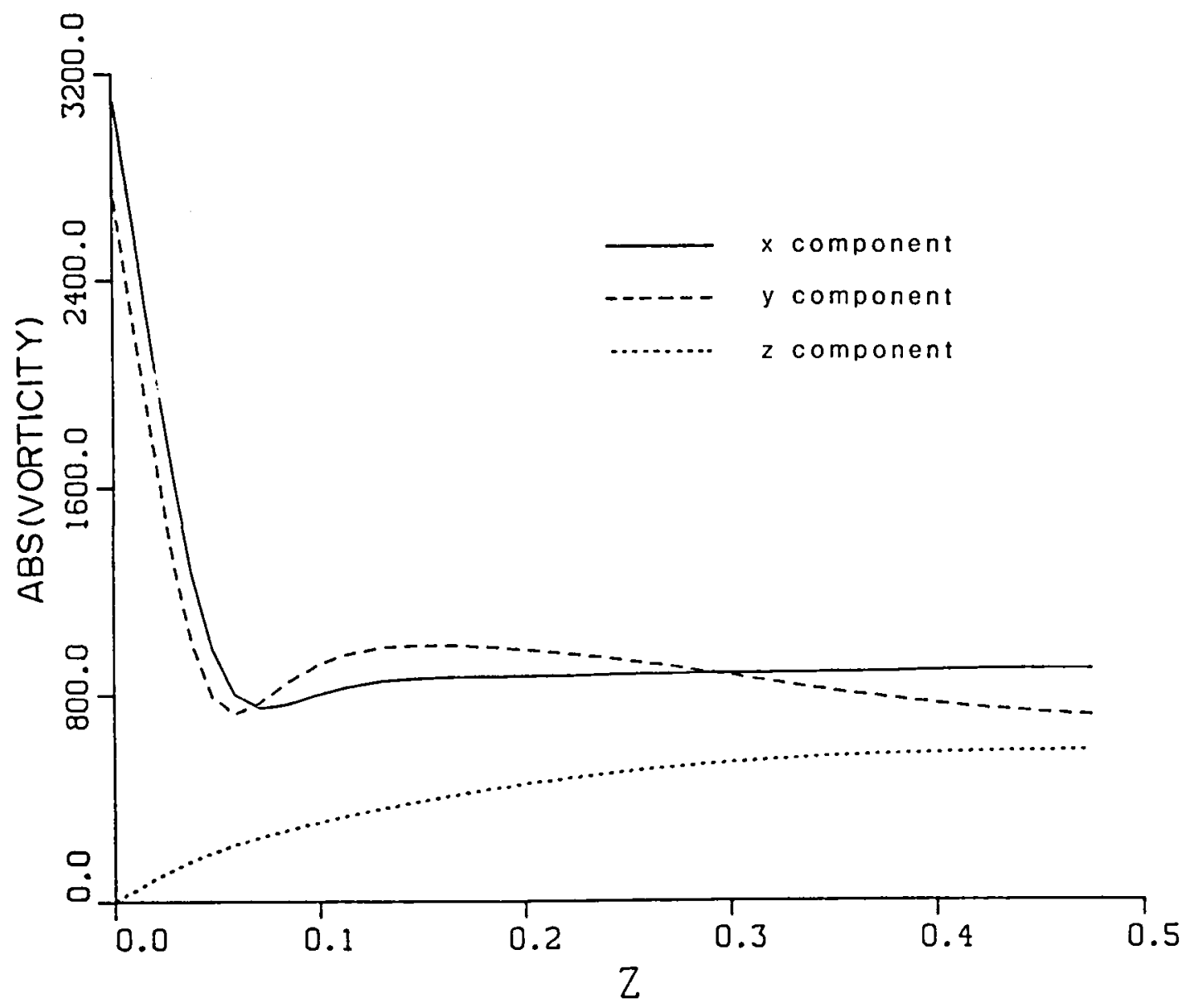

Figure 7. RMS of the three vorticity components versus height. 


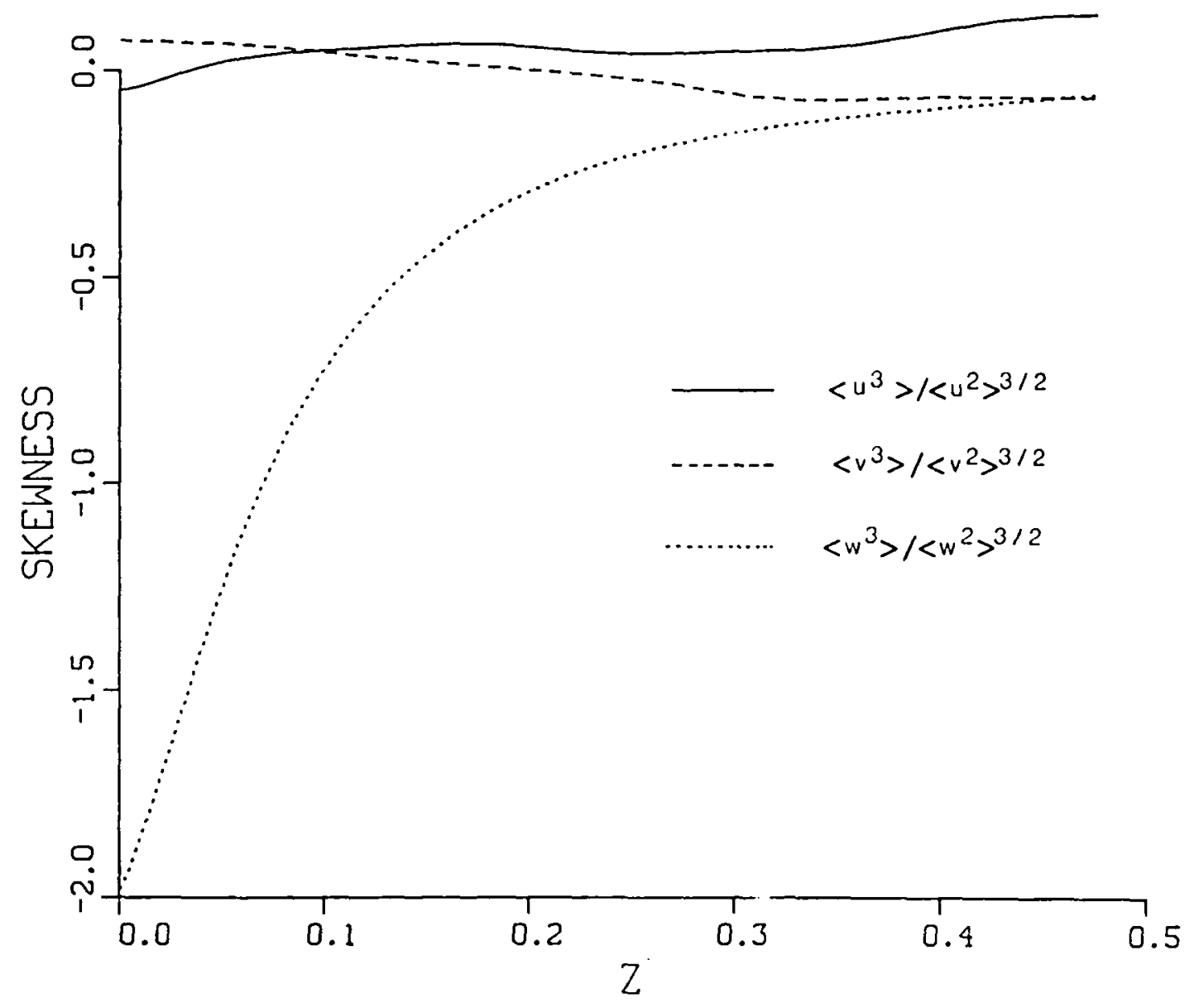

Figure 8. Skewness factor of the three velocity components versus height. 


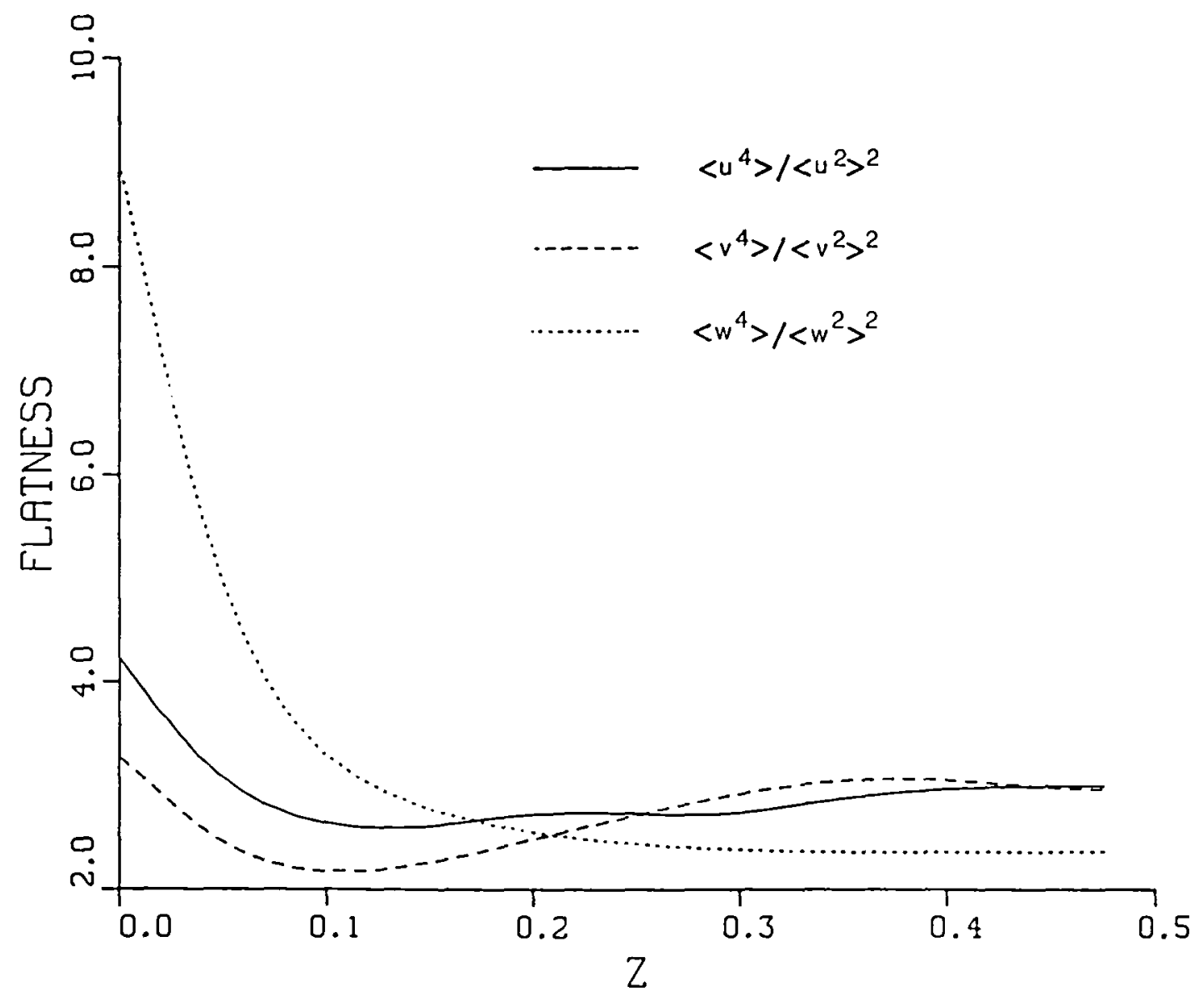

Figure 9. Flatness factor of the three velocity components versus height. 


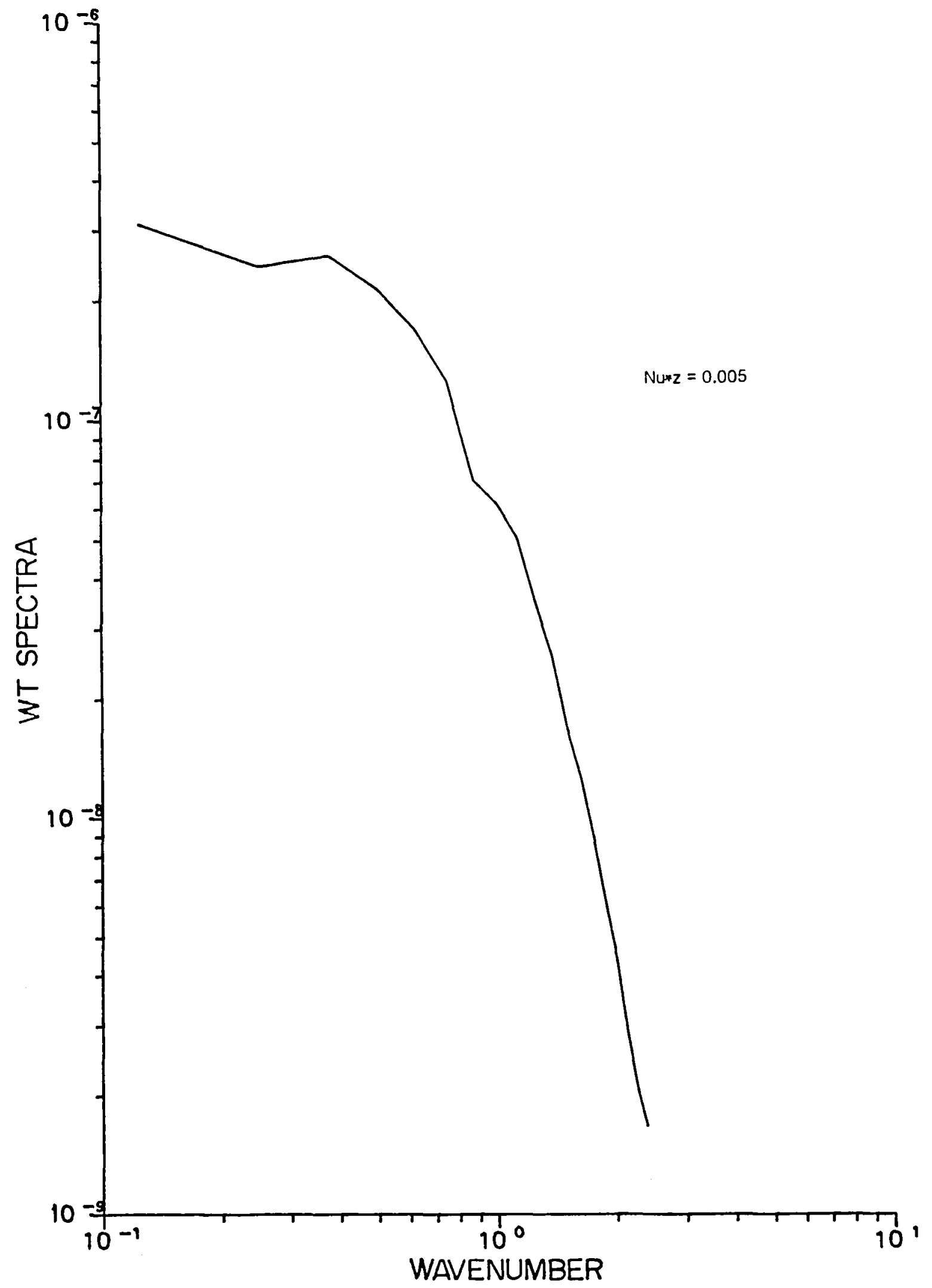

Figure 10. One-dimensional wT cross spectra (averaged in $y$ only) versus $x$ wavenumber. 


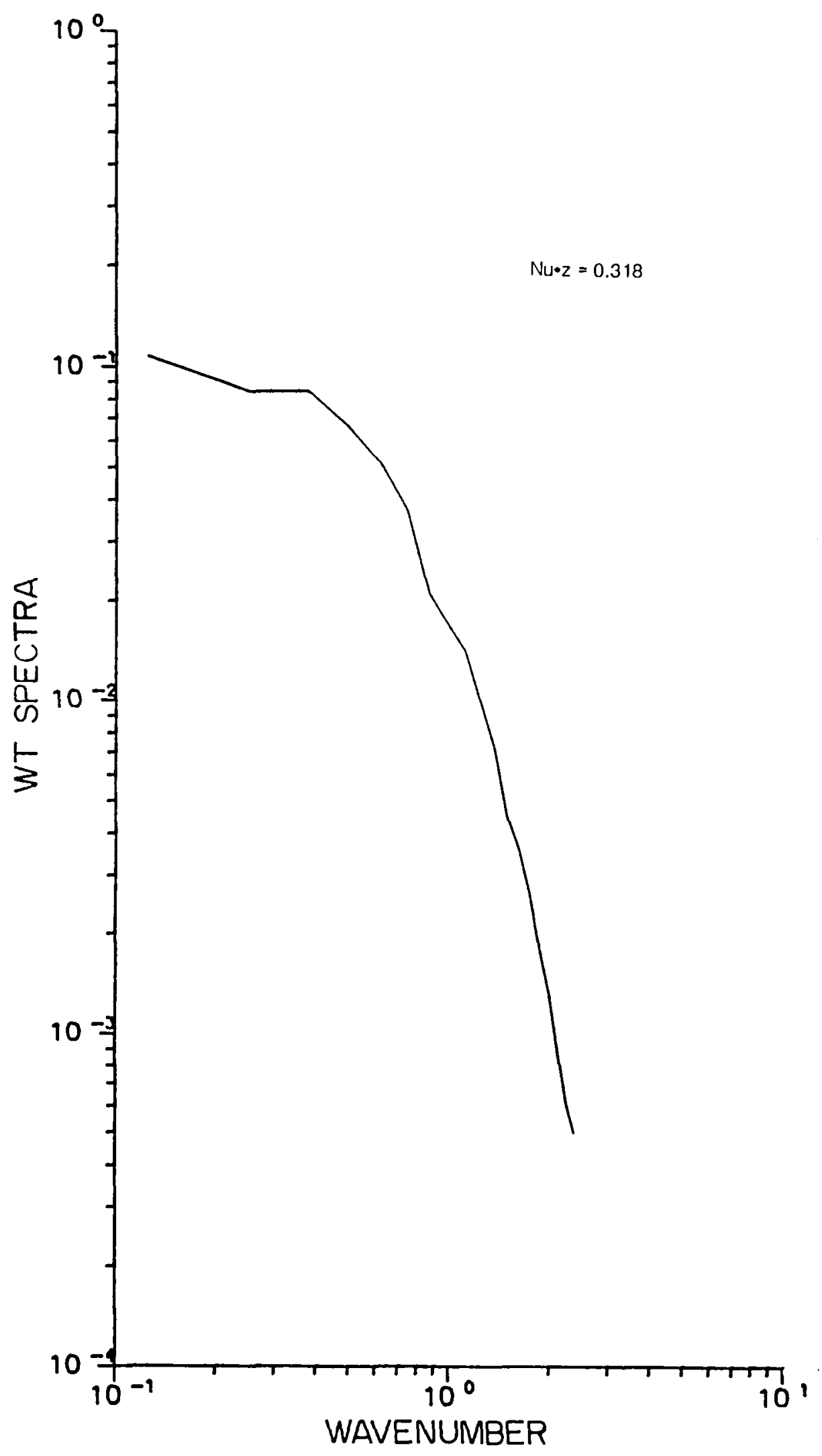

Figure 11. One-dimensional WT cross spectra (averaged in $y$ only) versus $x$ wavenumber. 


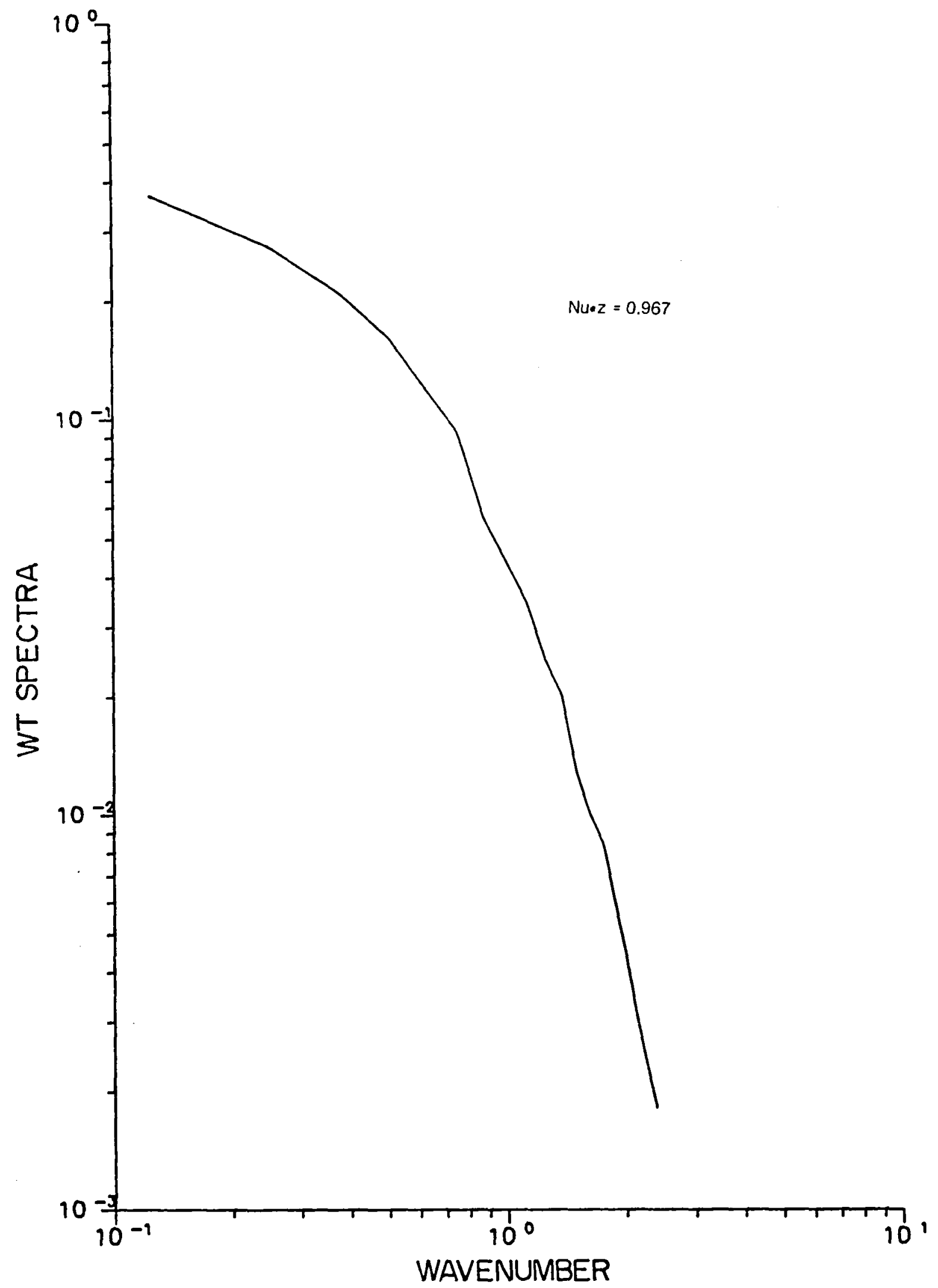

Figure 12. One-dimensional wT cross spectra (averaged in $y$ only) versus $x$ wavenumber. 


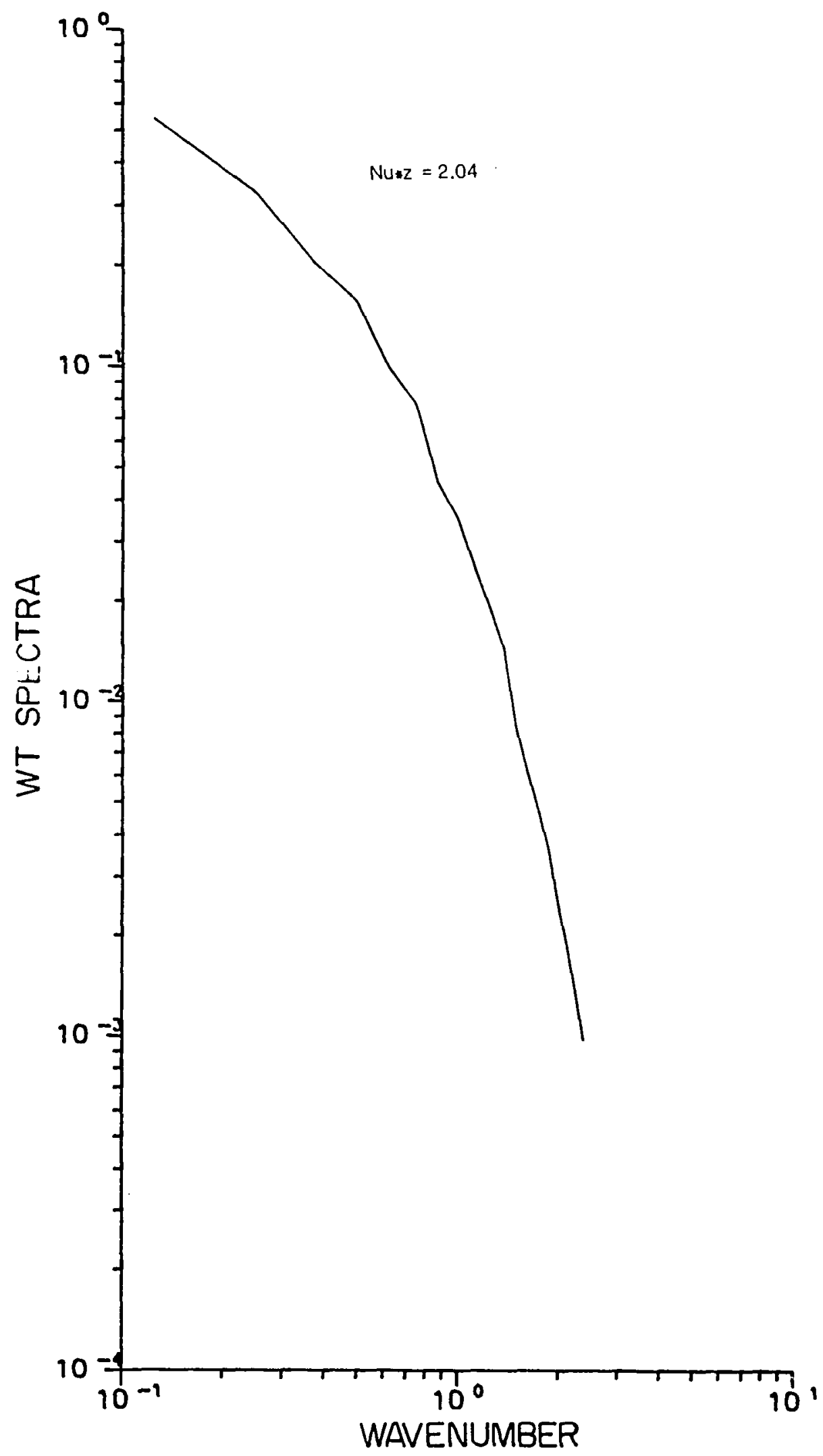

Figure 13. One-dimensional WT cross spectra (averaged in y only) versus $x$ wavenumber. 


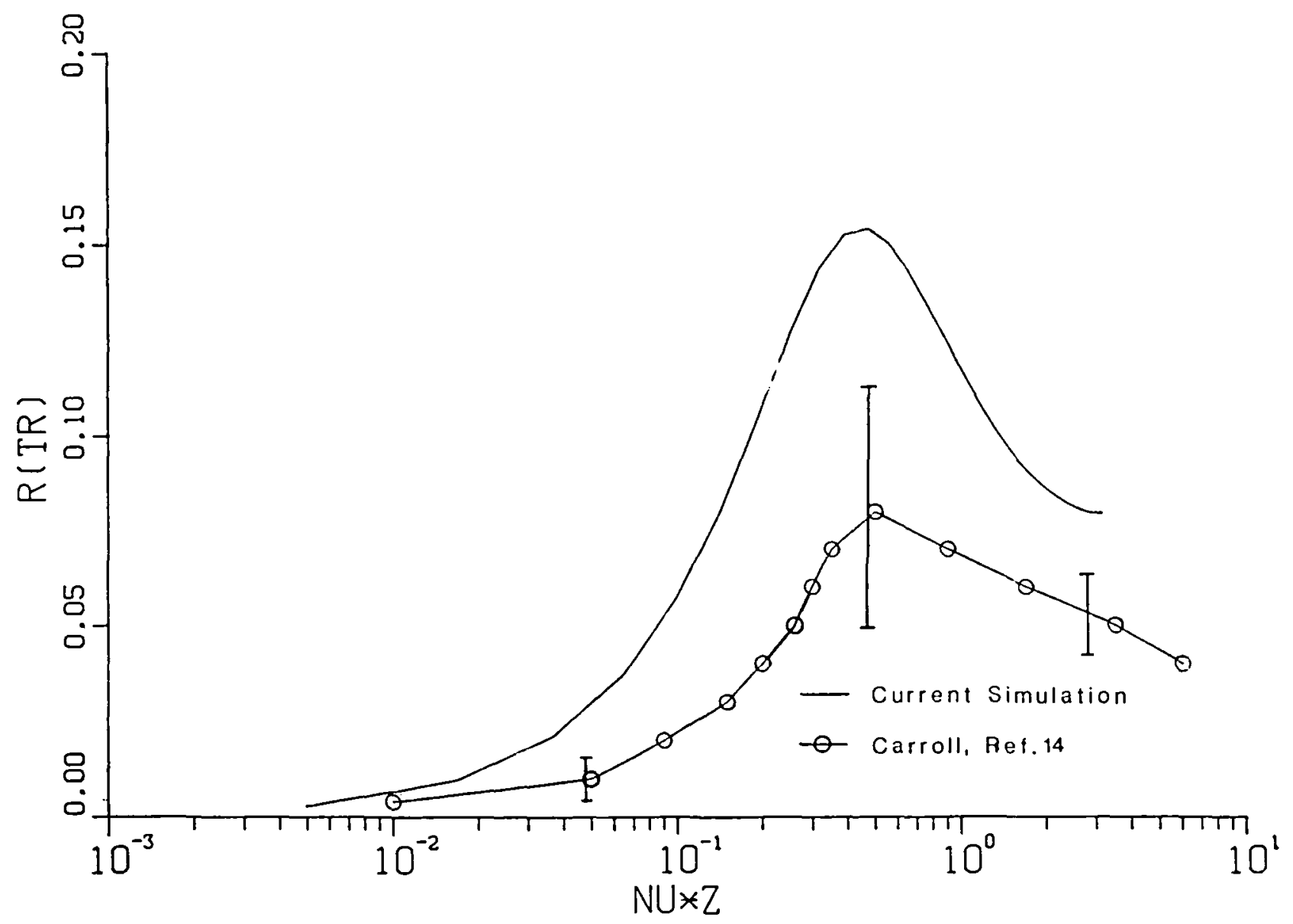

Figure 14. Comparison with experiment of the RMS relative temperature versus normalized height. 


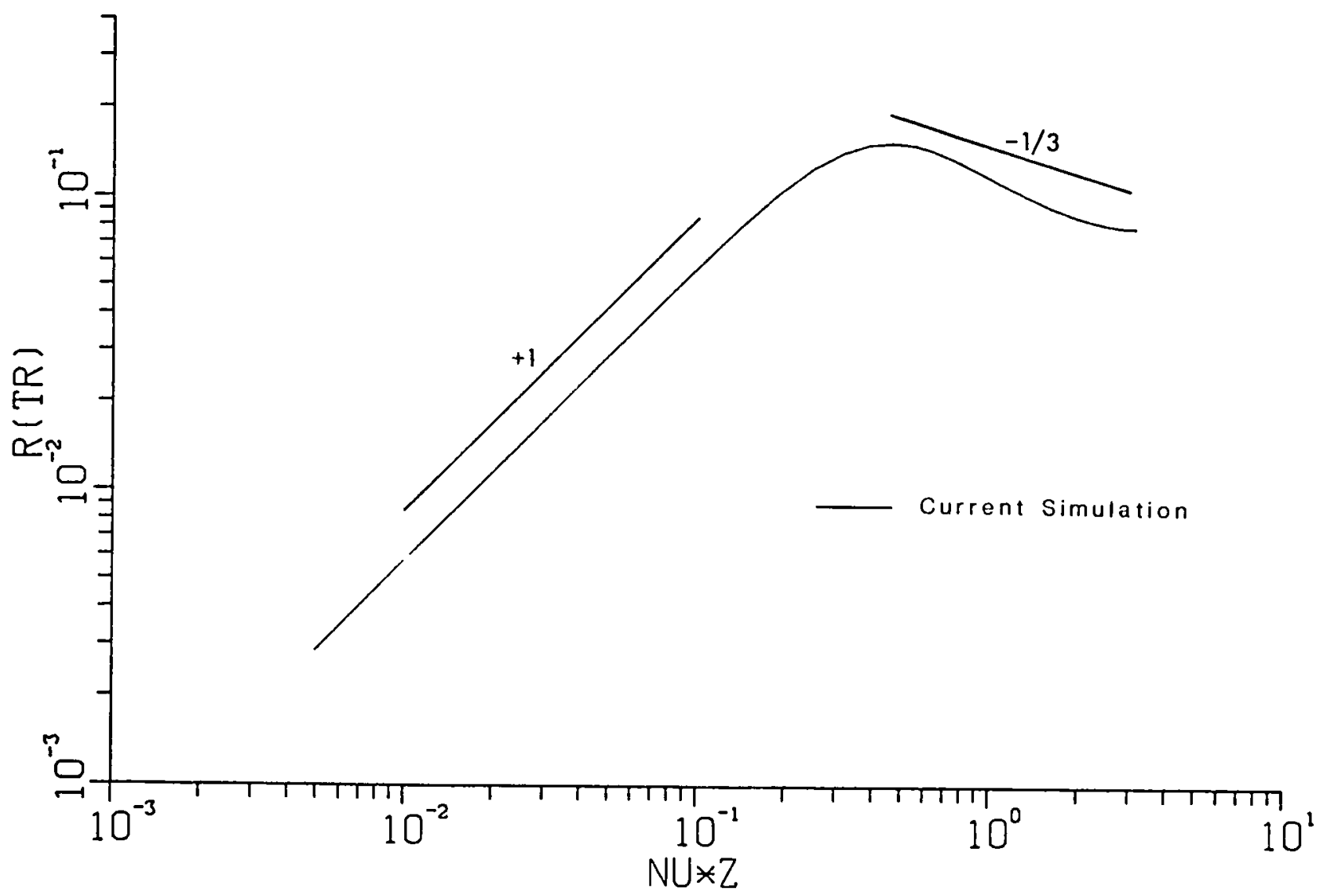

Figure 15. RMS relative temperature versus normalized height. The experimentally determined slopes of Carroll are shown for comparison. 


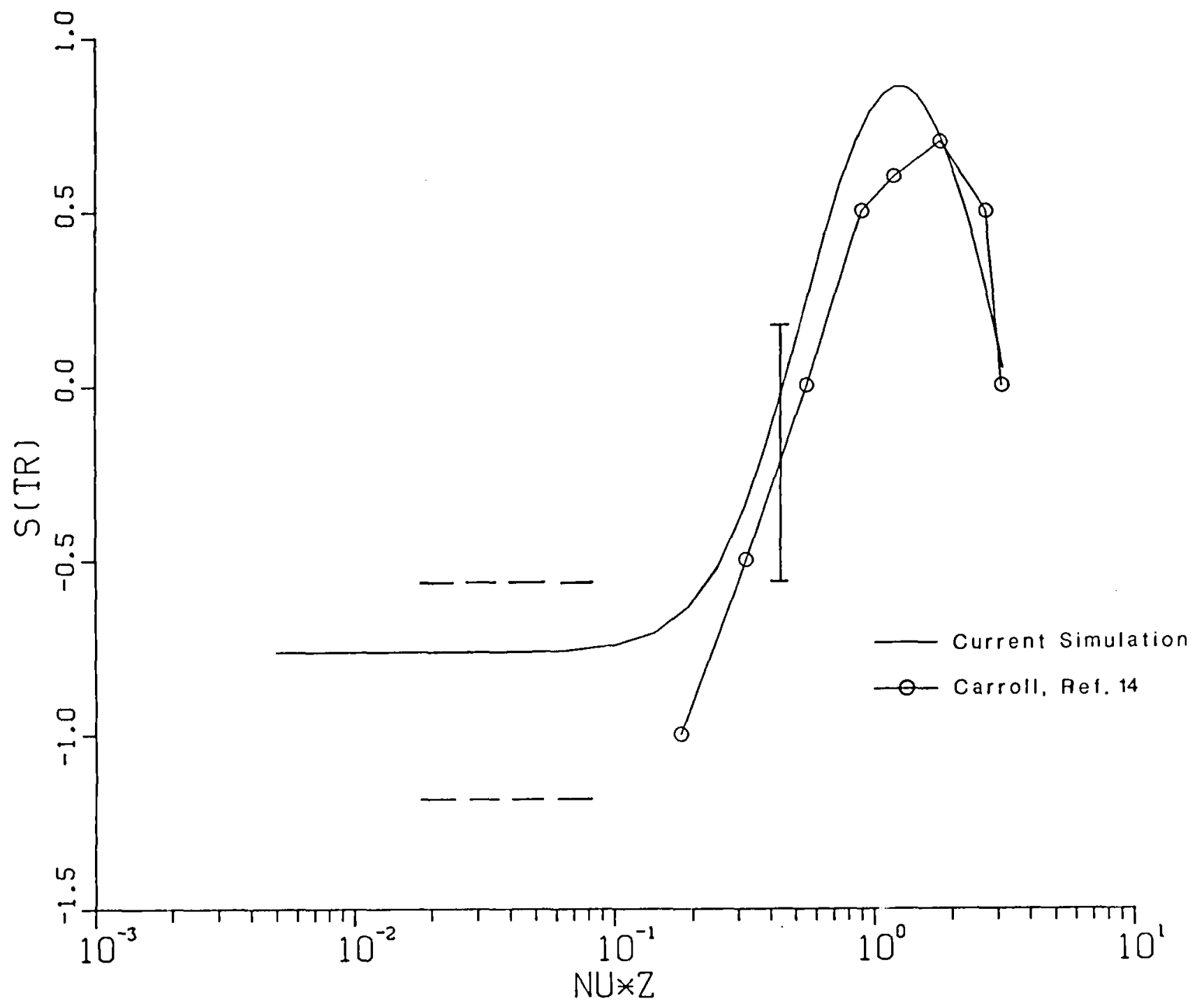

Figure 16. Comparison with experiment of the skewness of the relative temperature versus normalized height. 


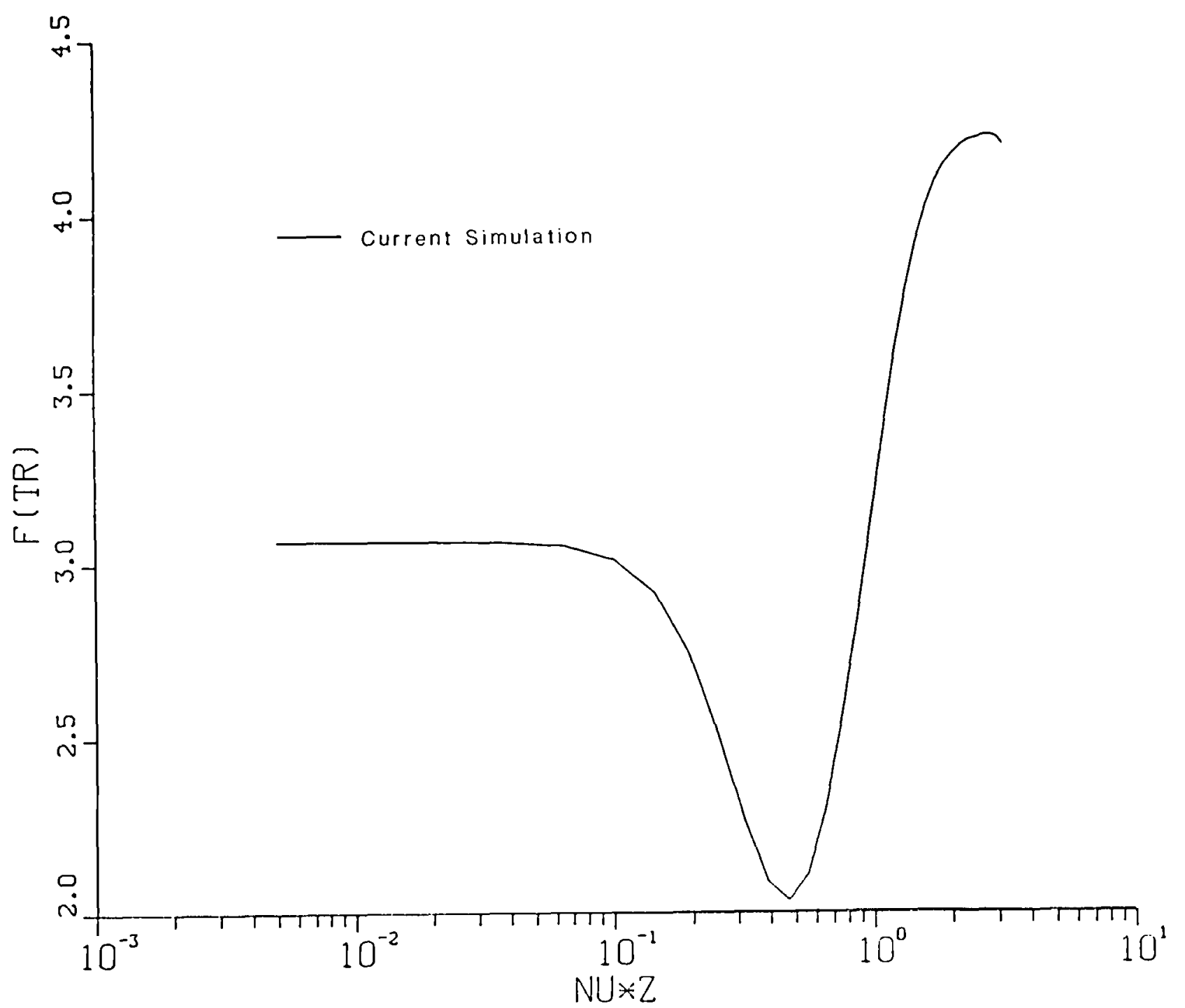

Figure 17. Flatness factor of the relative temperature versus normalized height. 


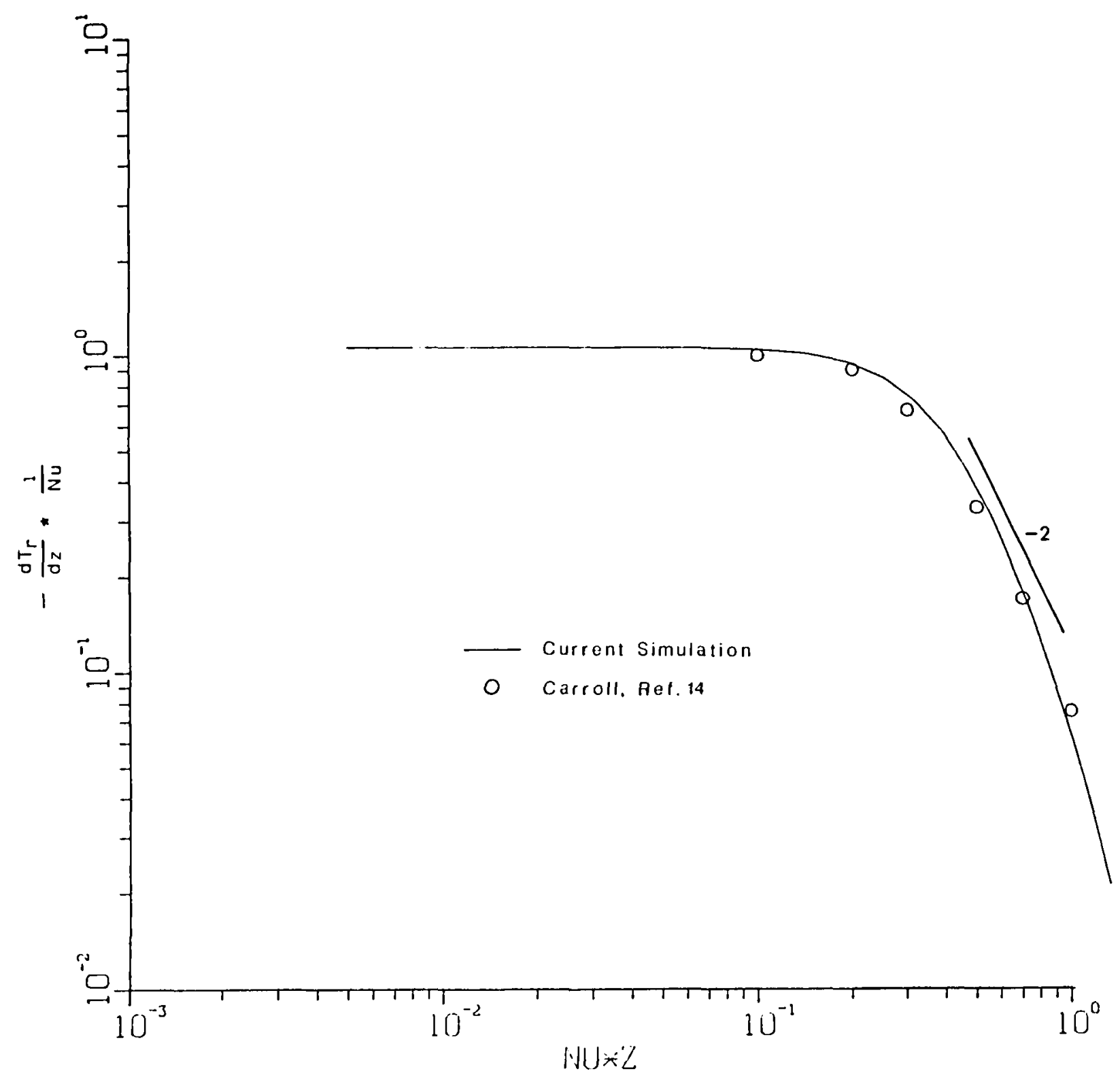

Figure 18. Comparison with experiment of the normalized relative temperature derivative versus norinalized height. The slope shown is from the theory of Malkus. 


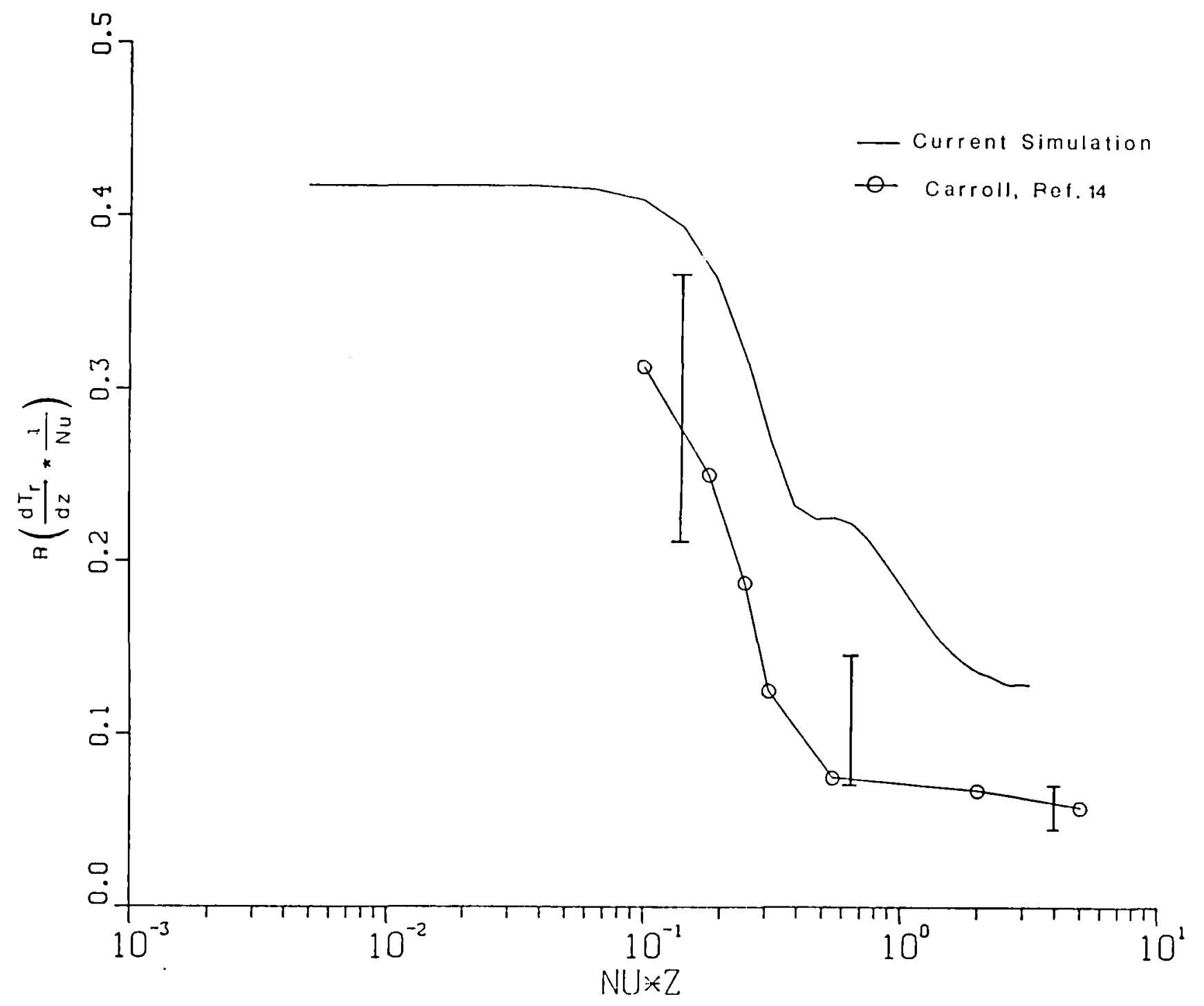

Figure 19. Comparisun with experiment of the RMS of the normalized relative temperature derivative. 


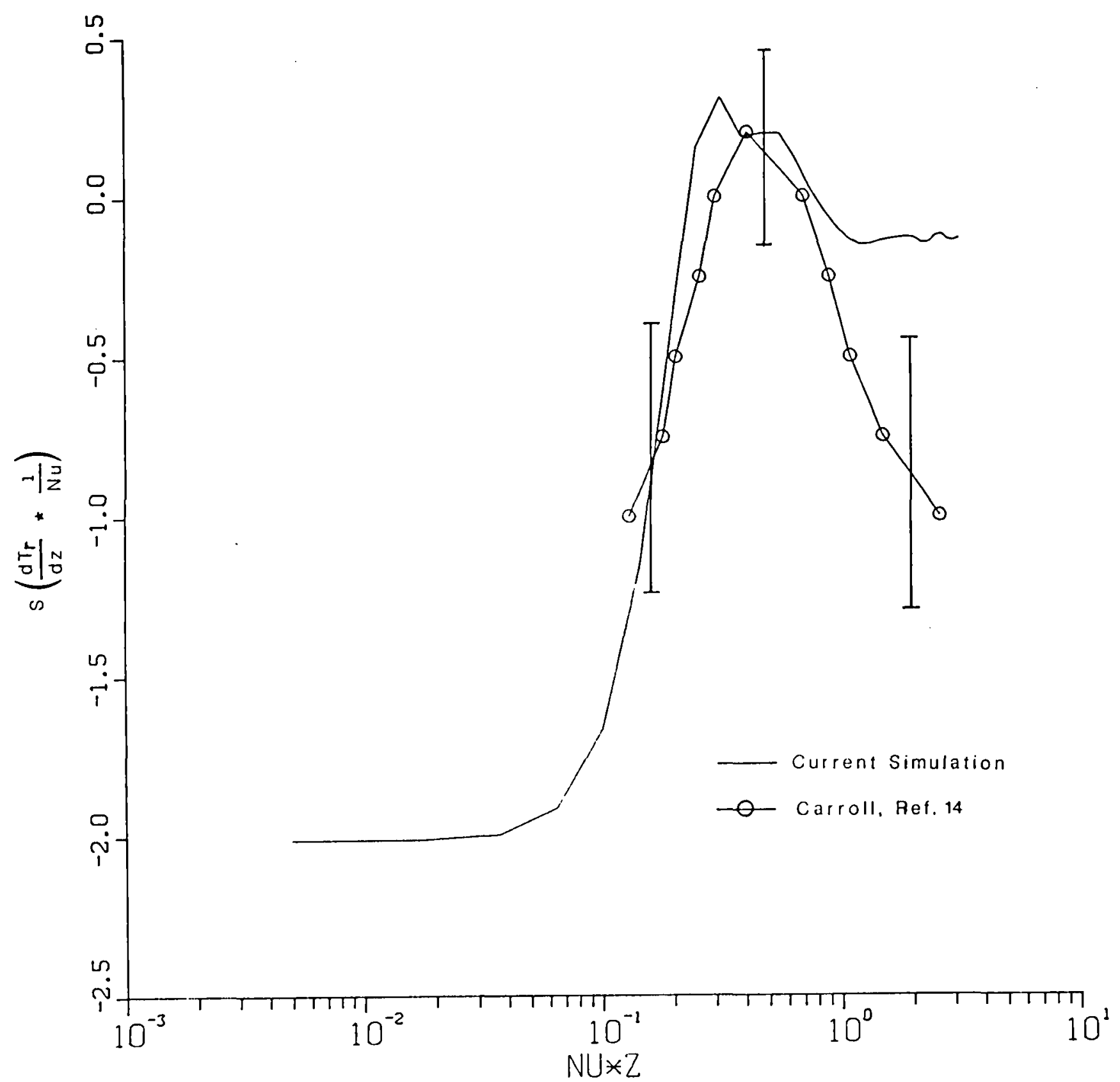

Figure 20. Comparison with experiment of the skewness factor for the normalized relative temperature derivative. 


\begin{tabular}{|c|c|c|}
\hline $\begin{array}{l}\text { 1. Report No. NASA CR-178027 } \\
\text { ICASE Report No. } 86-6\end{array}$ & 2. Government Accession No. & 3. Recipient's Catalog No. \\
\hline \multirow{2}{*}{\multicolumn{2}{|c|}{$\begin{array}{l}\text { 4. Title and Subtitle } \\
\text { SIMULATION OF THE TURBULENT RAYLEIGH-BENARD PROBLEM } \\
\text { USING A SPECTRAL/FINITE DIFFERENCE TECHNIQUE }\end{array}$}} & $\begin{array}{l}\text { 5. Report Date } \\
\text { February } 1986\end{array}$ \\
\hline & & 6. Performing Organization Code \\
\hline \multicolumn{2}{|c|}{$\begin{array}{l}\text { 7. Author(s) } \\
\text { T. M. Eidson, M. Y. Hussaini, and T. A. Zang }\end{array}$} & $\begin{array}{l}\text { 8. Performing Organization Report No. } \\
86-6\end{array}$ \\
\hline \multicolumn{2}{|c|}{$\begin{array}{l}\text { 9. Prforming Qranizatiog dappe agd Adpresications in Science } \\
\text { and Engineering } \\
\text { Mail Stop 132C, NASA Langley Research Center } \\
\text { Hampton, VA 23665-5225 }\end{array}$} & 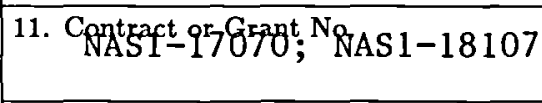 \\
\hline \multirow{2}{*}{\multicolumn{2}{|c|}{$\begin{array}{l}\text { 12. Sponsoring Agency Name and Address } \\
\text { National Aeronautics and Space Administration } \\
\text { Washington, D.C. } 20546\end{array}$}} & $\begin{array}{l}\text { 13. Type of Report and Period Covered } \\
\text { Contractor Report }\end{array}$ \\
\hline & & 14. Sponsoring Agency Code \\
\hline $\begin{array}{l}\text { 15. Supplementary Notes } \\
\text { Langley Technical Monitor: } \\
\text { J. C. South } \\
\text { Final Report }\end{array}$ & \multicolumn{2}{|c|}{$\begin{array}{l}\text { Proc. EUROMECH } 199 \text { Direct and Large } \\
\text { Eddy Simulation of Turbulent Flows, } \\
\text { Munich, Germany, } 9 / 30-10 / 2,1985 .\end{array}$} \\
\hline \multicolumn{3}{|l|}{ 16. Abstract } \\
\hline \multicolumn{3}{|c|}{$\begin{array}{l}\text { The three-dimensional, incompressible Navier-Stokes and energy equations } \\
\text { with the Bousinesq assumption have been directly simulated at a Rayleigh } \\
\text { number of } 3.8 \times 10^{5} \text { and a Prandt number of } 0.76 \text {. In the vertical direction, } \\
\text { wall boundaries were used and in the horizontal, periodic boundary conditions } \\
\text { were used. A spectral/finite difference numerical method was used to simulate } \\
\text { the flow. The flow at these conditions is turbulent and a sufficiently fine } \\
\text { mesh was used to capture all relevant flow scales. The results of the } \\
\text { simulation are compared to experimental data to justify the conclusion that } \\
\text { the small scale motion is adequately resolved. }\end{array}$} \\
\hline \multicolumn{3}{|l|}{$\begin{array}{l}\text { 17. Key Words (Suggested by Authors(s)) } \\
\text { Raleigh-Benard problem, } \\
\text { direct turbulence simulation }\end{array}$} \\
\hline $\begin{array}{l}\text { 19. Security Classif.(of this report) } \\
\text { Unclassified }\end{array}$ & $\begin{array}{l}\text { 20. Security Classif.(of this pag } \\
\text { Unclassif }\end{array}$ & \begin{tabular}{|l|l|} 
21. & No. of Pages \\
3
\end{tabular} \\
\hline
\end{tabular}


